\title{
金属有机框架质子导体及其质子交换膜的研究进展
}

\author{
孙炼王洪磊余金山周新贵* \\ (国防科技大学空天科学学院新型陶瓷纤维及其复合材料重点实验室 长沙 410073)
}

\begin{abstract}
摘要 质子交换膜是新型燃料电池的关键组件之一. 以 Nafion 为代表的商用全氟磺酸质子交换膜成本较高、操作温度 较低，限制了宽温度范围下的大规模应用. 金属有机框架材料(Metal Organic Framework, MOFs)因其比表面积大、结构 规整、可设计性强等优点, 在质子交换领域备受关注. 作者从三方面综述了 MOFs 质子导体的相关研究. 第一部分主 要介绍了 MOFs 传导质子的作用机理; 第二部分从有水/无水条件下工作的两种不同 MOFs 出发综述了 MOFs 质子导体 的相关发展; 第三部分系统回顾了 MOFs 质子交换膜的相关研究, 包括 MOFs 薄膜与 MOFs 混合基质膜结构. 最后指 出了 MOFs 质子导体及其质子交换膜研究中尚未解决的问题, 并展望该领域的未来研究方向.
\end{abstract}

关键词＼cjkstart金属有机框架; 质子导体; 质子交换膜; 燃料电池

\section{Recent Progress on Proton-Conductive Metal-Organic Frameworks and Their Proton Exchange Membranes}

\author{
Sun, Lian Wang, Honglei Yu, Jinshan Zhou, Xingui* \\ (Science and Technology on Advanced Ceramic Fibers and Composites Laboratory, College of Aerospace Science and \\ Engineering, National University of Defense Technology, Changsha 410073, China)
}

\begin{abstract}
Proton exchange membranes (PEMs) are important components for novel fuel cells. A significant effort has been made by researchers towards proton conductive materials and membranes, some of which have been successfully commercialized. However, commercial perfluorosulfonic acid membranes like Nafion suffer key issues which limit their large-scale applications in a wide temperature range, including high cost and low operation temperature. Therefore, it is highly desirable to prepare new-type PEMs possessing high proton conductivity, thermal and chemical stability, water uptake and excellent durability. Metal organic frameworks (MOFs) are attractive candidates for proton exchange membranes due to their high porosity, ordering pore structures and excellent designability. This review focuses on the recent progress on proton-conductive MOF structures and their proton exchange membranes. In the first section, the authors briefly introduce the proton conducting mechanism of MOFs and their testing methods. The Grotthuss mechanism refers to the proton transferring process in a continuous and long-range hydrogen network, whereas the Vehicular mechanism involves in the diffusion of proton carrier molecules. Then in the next section, the authors summarize the progress on bulk MOFs proton conductors. According to the work condition, proton-conducting MOFs can be divided into two types, namely working under humid and anhydrous environment. These works show the potential of proton-conductive MOFs to be applied in a wide temperature range, and some of them even have reached a relatively high conductivity larger than $10^{-2} \mathrm{~S} \cdot \mathrm{cm}^{-1}$, comparable with Nafion. In the third section, a review on the MOFs-based proton exchange membranes is shown. Researchers have proven that MOFs thin films have huge potential on proton conduction. Nevertheless, most of the MOFs-based PEMs are still mixed matrix membrane (MMM) structure. In order to boost the performance of MMMs-type MOFs-based PEMs, several strategies can be applied such as modifying MOF with functional groups, using 1D/2D MOFs structure and introducing the third phase into membranes. Last, the authors discuss the current issues and perspectives on MOFs proton conductors and their PEMs.
\end{abstract}

Keywords metal-organic frameworks; proton conductor; proton exchange membrane; fuel cells

\section{1 引言}

质子交换膜燃料电池具有效率高、容量大、寿命长、 低温启动速率快、环境友好等特点, 是目前最具发展前 景的动力系统之一 ${ }^{[1]}$, 对解决世界性的能源短缺与大气 污染问题有重要作用. 质子交换膜(Proton Exchange Membrane, PEMs)是质子交换膜燃料电池的重要组成部
分，用于高效传递质子，阻隔燃料泄漏等. 目前应用最 广泛的商用质子交换膜是以美国杜邦公司开发的 Nafion 系列为代表的全氟磺酸聚合物膜. 该种聚合物的 主链由疏水的四氟乙烯与全氟乙烯基醚聚合而成，而侧 链悬挂大量亲水的全氟磺酸基团 ${ }^{[2]}$. 但这种全氟磺酸膜 价格昂贵，高温下性能显著下降，且容易发生降解，严 重限制了其在复杂环境下的运行寿命, 特别是在高温质

\footnotetext{
*E-mail: zhouxinguilmy@163.com; Tel.: 0731-87007681

Received June 9, 2020; published July 30, 2020.

Project supported by the National Natural Science Foundation of China (Nos. 91426304, 51372274, 51502343).

项目受国家自然科学基金(Nos. 91426304, 51372274, 51502343)资助.
} 
子交换膜燃料电池(运行温度 $120 \sim 200{ }^{\circ} \mathrm{C}$ )方面的应用. 开发新型 PEMs 一直是该领域的研究热点. 具体而言, 新型 PEMs 应具备成本低廉、好的热稳定性和化学稳定 性、较高保水性、优异力学性能、高选择性(质子电导 率与燃料渗透率的比值) 、在宽温域下保持良好质子电 导率等特点 ${ }^{[3-5]}$.

金属有机框架材料 (Metal-Organic Frameworks, $\mathrm{MOFs}$ ) 是一种由含金属(金属离子或金属簇)单元同有机 配体通过强作用力结合而成的孔隙率高、内部通道丰富 的有机-无机杂化材料. 通过调控金属与有机配体的种 类与配位方式, MOFs 可以呈现特定的结构与性能, 这 使其广泛应用于催化、传感、气体储存与分离等重要领 域 ${ }^{[6-9]}$. 关于 MOFs 质子导体的研究开始于 1979 年, Kanda 等 ${ }^{[10]}$ 首次通过粉末压片法证实了 $\left(\mathrm{HOC}_{2} \mathrm{H}_{4}\right)_{2}-\mathrm{dtoa}-\mathrm{H}_{2}$ 二维结构的质子导电性能. 此后, 人 们开始认识到 MOFs 作为质子导体的潜力, 并不断设 计、开发具有优异质子传递能力的新型 MOFs 结构. MOFs 应用于质子传递领域的优势主要有 ${ }^{[11]}$ : (1) MOFs 的多孔特性, 使得其能够有效吸附质子载体, 提供质子 传递的有效活性位点; (2) MOFs 的孔道结构规整性, 使 得其内部形成了质子快速传递的有效路径; (3) MOFs 的 合成简便, 使得大规模制备符合特定需求的质子导体成 为可能. 而如何将 MOFs 转化为膜结构是其应用于燃料 电池隔膜系统的关键. 目前, 虽然已有关于 MOF 质子 导体的综述 ${ }^{[12-14]}$, 但是仍缺少对 MOFs 基质子交换膜结 构相关的系统总结. 本综述主要针对近年来国内外对新 型 MOFs 质子导体及其质子交换膜的研究进行总结论 述，并对未来其发展进行展望(图 1).

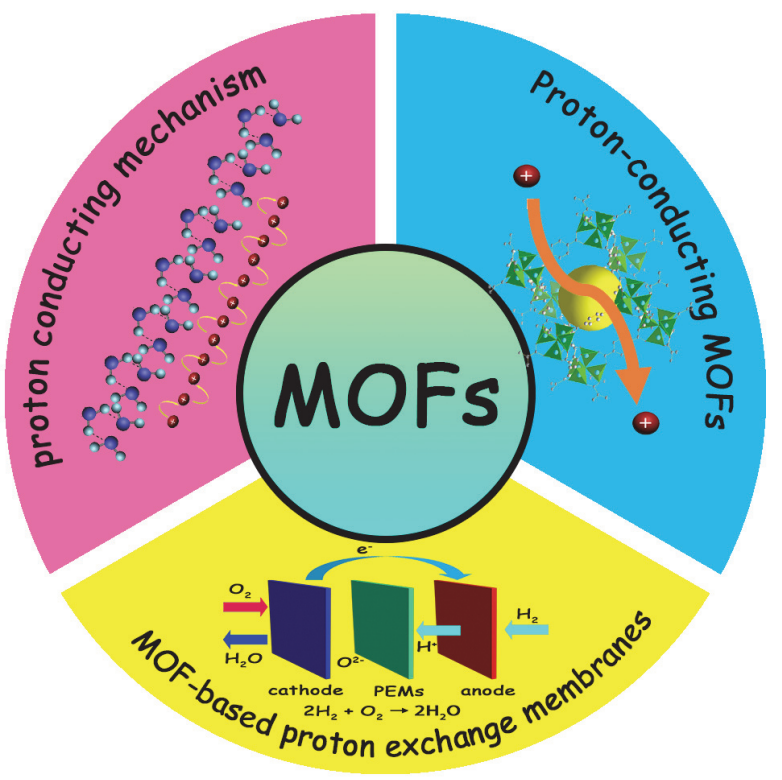

图 $1 \mathrm{MOFs}$ 质子导体及其质子交换膜的研究重点

Figure 1 Research toolbox for proton-conducting MOFs and their membranes

\section{MOFs 的质子导电机理}

研究者们广泛认为 MOFs 传递质子主要依靠两种 机制: 质子跳跃机制(Grotthuss mechanism)与质子运输 机制(Vehicular mechanism)(图 2) ${ }^{[15,16]}$. 质子跳跃机制的 过程为: 首先，质子导体内部相邻的分子或基团形成大 规模的氢键网络; 而后质子通过与水分子结合形成水合 氢离子; 最后, 在化学势的驱动下，质子通过氢键网络 跳跃到下一个邻近的分子或基团，完成了质子的迁移和 传递. 质子运输机制则是依靠携带质子的载体在导体内 部的扩散完成传输过程. 在该过程中, 携带质子的载体 沿传输方向运动, 这些载体扩散完成后会在导体内形成 浓度梯度, 使剩余未携带质子的载体沿反方向运动. 质 子跳跃机制仅涉及质子的转移, 所需活化能 $\left(E_{\mathrm{a}}\right)$ 一般小 于 $0.4 \mathrm{eV}^{[17]}$; 而质子运输机制涉及体积较大的质子载体 的扩散, 所需活化能较大 $\left(E_{\mathrm{a}}>0.4 \mathrm{eV}\right)^{[3]}$. 值得一提的是, 由于实际质子导体的运行环境较为复杂, 所以质子传输 常常依靠两种机制结合的方式进行.

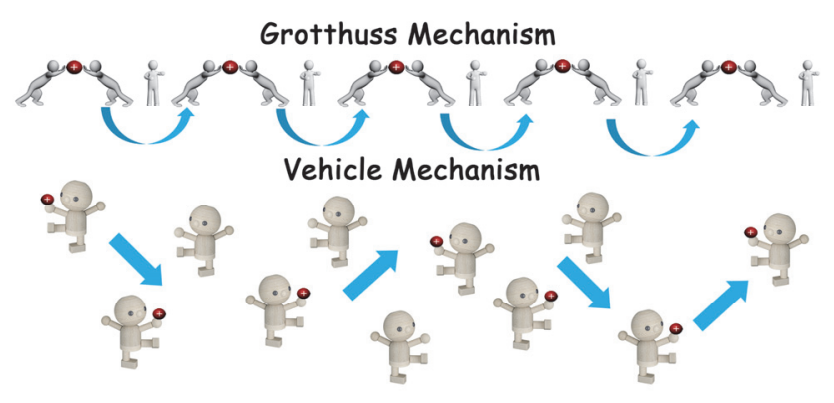

图 2 质子传递中跳跃机制与运输机制的示意图

Figure 2 Schematic illustration of Grotthuss and Vehicle mechanism in proton conducting

常用研究MOFs 的质子传导能力的方法是交流阻抗 法. 此方法一般将合成的 MOFs 粉末压成一定大小和厚 度的圆片进行电化学测试，结合其它表征手段分析 MOFs 质子导体的作用机理 ${ }^{[18]}$. 然而此法测试得到的 MOFs 电导率受晶界的影响较大. 为了细致研究 MOFs 质子导电的本质，更多人采用单晶测量法. 单晶法的最 大优势是能够测量 MOFs 晶体各方向的质子传输性能, 使得人们认识到 MOFs 的质子导电具有各向异性. Kitagawa 等 ${ }^{[19]}$ 制备了一种二维结构配位框架化合物 $\mathrm{Zn}\left(\mathrm{H}_{2} \mathrm{PO}_{4}\right)_{2}(\mathrm{TzH})_{2}$ (图 3a). 这种 $\mathrm{MOFs}$ 的无水质子电导 率为 $1.51 \times 10^{-3} \mathrm{~S} \cdot \mathrm{cm}^{-1}\left(150{ }^{\circ} \mathrm{C}, E_{\mathrm{a}}=0.6 \mathrm{eV}\right)$, 并且单晶 测试结果表明(图 3b)该晶体平行于 $a b$ 平面方向的电导 率远高于垂直于 $a b$ 面的 $c$ 方向, 证明 $a b$ 平面的氢键网 络是该 MOFs 质子导电性的来源. 除此之外, 准弹性电

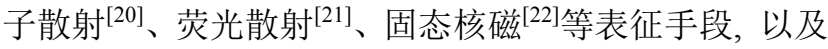
分子动力学模拟 ${ }^{[23,24]}$ 、密度泛函理论 ${ }^{[2]}$ 等分析计算技术 使得直观了解质子在 MOFs 中的运动成为可能. 


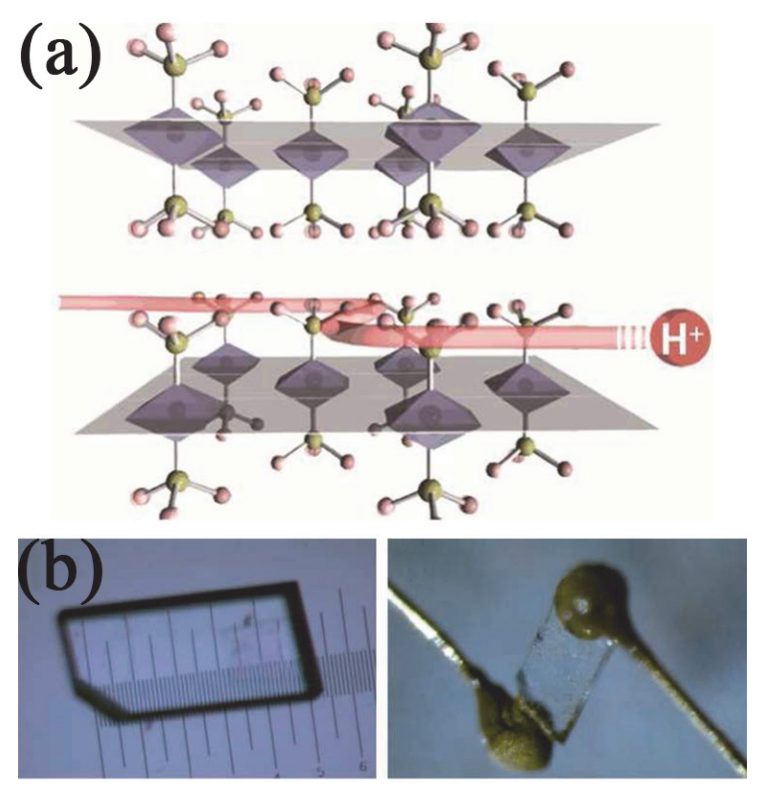

图 3 (a) $\mathrm{Zn}\left(\mathrm{H}_{2} \mathrm{PO}_{4}\right)_{2}(\mathrm{TzH})_{2}$ 的结构示意图; (b) $\mathrm{Zn}\left(\mathrm{H}_{2} \mathrm{PO}_{4}\right)_{2}(124 \mathrm{triH})_{2}$ 的 单晶结构(左)及单晶电导率测试方法(右) ${ }^{[19]}$

Figure 3 (a) Structure schematic illustration, (b) single crystal structure (left) and its testing method with gold electrode (right) of $\mathrm{Zn}\left(\mathrm{H}_{2} \mathrm{PO}_{4}\right)_{2}(\mathrm{TzH})_{2}{ }^{[19]}$

\section{3 基于 MOFs 的质子导体}

根据工作条件的不同, MOFs 质子导体可以分类为 在有水条件工作的质子导体, 以及在无水条件下工作的 质子导体. 前者必须在低温条件下 $\left(<100{ }^{\circ} \mathrm{C}\right)$ 工作, 因 为质子的传输主要依靠水分子搭建的氢键网络进行; 而 温度高于 $100{ }^{\circ} \mathrm{C}$ 时, 常需要向 $\mathrm{MOFs}$ 孔道负载携带质子 的高沸点客体完成质子传递过程. 目前, 提高 MOFs 质 子电导率的常用策略有: (1)通过一步法或后处理方法在 MOFs 配体上修饰特定官能团(如草酸盐、磺酸盐、羧酸 盐、膦酸盐等)作为质子受体; (2)通过负载相应的质子载 体同 MOFs 骨架形成完整的质子传递路径; (3)结合以上 两种策略共同提高 MOFs 的质子导电能力. 表 1 总结了
近年来部分代表性 MOFs 质子导体的性能.

\section{1 有水条件下工作的 MOFs 质子导体}

在水媒介下, MOFs的骨架和孔径率决定了其吸水 能力, 进而影响质子传递网络的结构. Sun 等 ${ }^{[26]}$ 采用水 热法合成了一种叠层状 MOF $[\mathrm{Cu}(p-\mathrm{IPhHIDC})]_{n}$. 这种 MOFs 在 $100{ }^{\circ} \mathrm{C}$ 与 $98 \%$ 相对湿度(Relative Humidity, $\mathrm{RH}$ ) 的电导率达到 $1.51 \times 10^{-3} \mathrm{~S} \cdot \mathrm{cm}^{-1}$. 该MOFs 在 $68 \%$ 和 $98 \%$ RH下对应的活化能分别为 1.79 与 $0.25 \mathrm{eV}$, 表明低湿和 高湿条件下分别符合Vehicular和Grotthuss传输机制. 他 们发现在稀薄水蒸气条件下样品的吸水量为 $75 \mathrm{mg} \cdot \mathrm{g}^{-1}$, 而蒸气压升高时样品的吸水量达到 $222 \mathrm{mg} \cdot \mathrm{g}^{-1}$, 证明了 较高的水吸附量使得该MOFs内部有效形成了氢键网络, 使得活化能大大降低, 改变了质子传递的方式.

在 MOFs 配体负载布仑斯惕酸(Brønsted Acid)官能 团是最常用的一种提升质子传递能力的策略. Kitagawa 组细致研究了金属-酸性基团配位结构的 MOFs 质子导 体. 2009 年, 由他们 ${ }^{[27]}$ 合成的一种草酸配位化合物 $\left[\mathrm{Fe}\left(\mathrm{C}_{2} \mathrm{O}_{4}\right)\left(\mathrm{H}_{2} \mathrm{O}\right)_{2}\right]$ 引领了近年对 MOFs 质子导体研究的 热潮(图 4a). 该化合物内部 $\mathrm{Fe}^{\mathrm{II}}$ 中心离子在赤道面同草 酸基团相连接成一维链状结构, 且同两个水分子形成配 位. 这些水分子沿链段方向排列, 形成了质子传递通道. 在室温与 $98 \% \mathrm{RH}$ 条件下, 其质子电导率达到了 $1.3 \times$ $10^{-3} \mathrm{~S} \cdot \mathrm{cm}^{-1}$, 对应活化能为 $0.37 \mathrm{eV}$. 这项工作证实了 MOFs 内部的水分子对其质子传递起到至关重要的作 用. 2011 年, 他们 ${ }^{[39]}$ 以含铁苯二羧酸盐为配体合成了柔 性 MOFs 结构 MIL-53(Fe)- $(\mathrm{COOH})_{2}$ (图 4b), 其质子传递 活化能低至 $0.21 \mathrm{eV}$. 他们认为体系中未配位的羧酸基 团的存在使溶液体系内的质子浓度升高, 大大增强质子 导电能力. 最近, 他们 ${ }^{[40]}$ 又使用尿素为配体合成出在室 温下电导率达到 $2.64 \times 10^{-2} \mathrm{~S} \cdot \mathrm{cm}^{-1}(95 \% \mathrm{RH})$ 的 MOF-74(Mg)-Urea- $d_{4}$ 结构(图 4c). 尿素分子的存在减少 了结构中的空隙，固定了结晶水分子，同时尿素分子在 水媒介中的极化作用也使其参与氢键网络的构建.

表 1 近年来(2017 2020 年)报道的部分高性能 MOFs 质子导体的关键性能

Table 1 Key properties of some reported high-performance proton-conductive MOFs in recent three years (2017 2020)

\begin{tabular}{|c|c|c|c|c|c|}
\hline MOF/Coordination polymer & Pore guests & $\begin{array}{l}\text { Conductivity/ } \\
\left(\mathrm{S} \bullet \mathrm{cm}^{-1}\right)\end{array}$ & Work condition & $E_{\mathrm{a}} / \mathrm{eV}$ & Ref. \\
\hline$\left(\mathrm{N}_{2} \mathrm{H}_{5}\right)\left[\mathrm{CeEu}\left(\mathrm{C}_{2} \mathrm{O}_{4}\right)_{4}\left(\mathrm{~N}_{2} \mathrm{H}_{5}\right)\right] \bullet 4 \mathrm{H}_{2} \mathrm{O}$ & $\mathrm{H}_{2} \mathrm{O}$ molecules & $3.42 \times 10^{-3}$ & Room temperature, $100 \% \mathrm{RH}$ & 0.10 & {$[21]$} \\
\hline BUT-8(Cr)A & $\mathrm{H}_{2} \mathrm{O}$ molecules & $1.27 \times 10^{-1}$ & $80{ }^{\circ} \mathrm{C}, 100 \% \mathrm{RH}$ & 0.11 & {$[28]$} \\
\hline$\left\{\left[\mathrm{Gd}_{2}\left(\mathrm{CO}_{3}\right)(\mathrm{ox})_{2}\left(\mathrm{H}_{2} \mathrm{O}\right)_{2}\right] \cdot 3 \mathrm{H}_{2} \mathrm{O}_{n}\right.$ & $\mathrm{H}_{2} \mathrm{O}$ molecules and oxalate & $1.98 \times 10^{-3}$ & $150{ }^{\circ} \mathrm{C}$, anhydrous & 0.27 & [29] \\
\hline$[\mathrm{Co}(\mathrm{DCDPP})] \cdot 5 \mathrm{H}_{2} \mathrm{O}$ & Carboxylic groups & $3.90 \times 10^{-2}$ & $80{ }^{\circ} \mathrm{C}, 97 \% \mathrm{RH}$ & 0.34 & {$[30]$} \\
\hline$\left\{\left[\mathrm{Tb}_{4}(\mathrm{TTHA})_{2}\left(\mathrm{H}_{2} \mathrm{O}\right)_{4}\right] \cdot 7 \mathrm{H}_{2} \mathrm{O}\right\}_{n}$ & Free $\mathrm{H}_{2} \mathrm{O}$ molecules & $2.57 \times 10^{-2}$ & $60{ }^{\circ} \mathrm{C}, 98 \% \mathrm{RH}$ & 0.68 & [31] \\
\hline Im-Fe-MOF & $\begin{array}{l}\text { Imidazone and coordinated } \\
\mathrm{H}_{2} \mathrm{O}\end{array}$ & $1.21 \times 10^{-2}$ & $60{ }^{\circ} \mathrm{C}, 98 \% \mathrm{RH}$ & 0.573 & {$[32]$} \\
\hline$\left.\left[\mathrm{ImH}_{2}\right] \mathrm{C} \mathrm{Cu}\left(\mathrm{H}_{2} \mathrm{PO}_{4}\right)_{2} \mathrm{Cl}\right] \cdot \mathrm{H}_{2} \mathrm{O}$ & Protonated imidazole & $2.00 \times 10^{-2}$ & $130{ }^{\circ} \mathrm{C}$, anhydrous & 1.10 & {$[33]$} \\
\hline$\left.\left\{\left[\mathrm{In}_{3} \mathrm{OL}_{1.5}\left(\mathrm{H}_{2} \mathrm{O}\right)_{3}\right]\left(\mathrm{NO}_{3}\right)\right\} \bullet(\mathrm{DMA})_{3} \bullet\left(\mathrm{CH}_{3} \mathrm{CN}\right)_{6} \bullet\left(\mathrm{H}_{2} \mathrm{O}\right)_{30}\right\}_{n}$ & Lattice $\mathrm{H}_{2} \mathrm{O}$ molecules & $1.25 \times 10^{-3}$ & $90{ }^{\circ} \mathrm{C}$, anhydrous & 0.45 & [34] \\
\hline$\left[\left(\mathrm{Cu}_{4}{ }_{4} \mathrm{Cu}_{4}{ }_{4}^{\mathrm{II}} \mathrm{L}_{4}\right) \cdot 3 \mathrm{H}_{2} \mathrm{O}\right]_{n}$ & $\mathrm{NH}_{3}$ and crystalline $\mathrm{H}_{2} \mathrm{O}$ & $1.13 \times 10^{-2}$ & $100{ }^{\circ} \mathrm{C}, 98 \% \mathrm{RH}$ & 0.37 & {$[35]$} \\
\hline SA-EIMS@MIL-101 & SA and EIMS & $1.89 \times 10^{-3}$ & $150{ }^{\circ} \mathrm{C}$, anhydrous & 0.326 & {$[36]$} \\
\hline $\mathrm{Zn}_{3}(\mathrm{bpdc})_{2}(\mathrm{pdc})(\mathrm{DMF}) \cdot 6 \mathrm{DMF}$ & $\mathrm{H}_{2} \mathrm{O}$ molecules & $0.95 \times 10^{-2}$ & $60{ }^{\circ} \mathrm{C}, 97 \% \mathrm{RH}$ & 0.45 & {$[37]$} \\
\hline$\left(\mathrm{NH}_{4}\right)_{3}\left[\mathrm{Zr}\left(\mathrm{H}_{2 / 3} \mathrm{PO}_{4}\right)_{3}\right]$ & $\mathrm{NH}_{4}^{+}$and phosphate pairs & $1.45 \times 10^{-3}$ & $180{ }^{\circ} \mathrm{C}$, anhydrous & 0.30 & [38] \\
\hline
\end{tabular}

${ }^{a} \mathrm{~N} / \mathrm{A}$ : 文中未提及(Not available). 

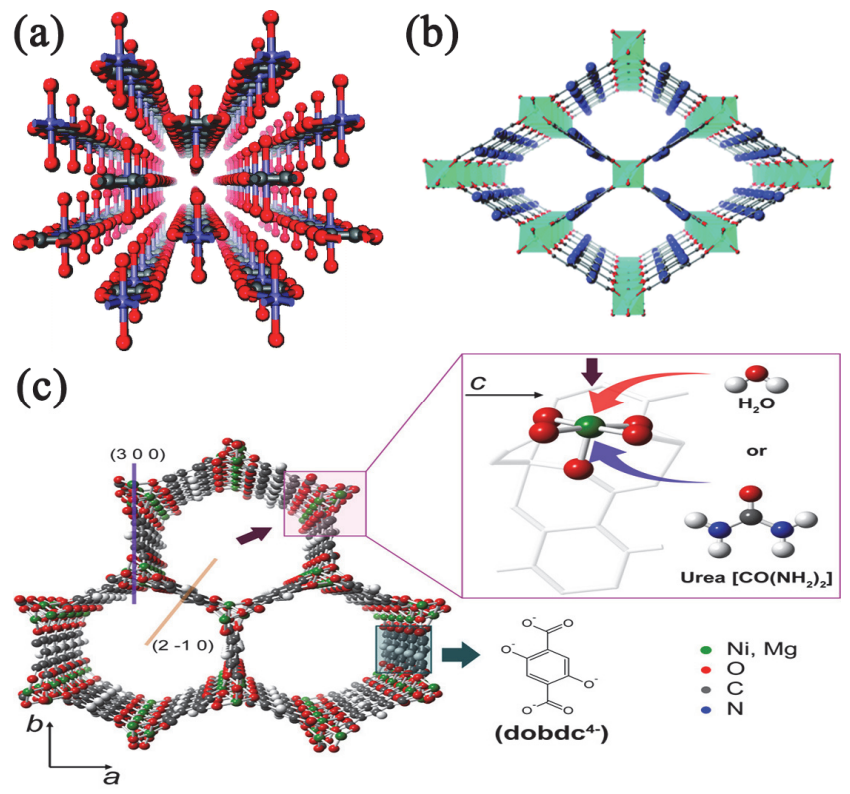

图 4 (a) $\left[\mathrm{Fe}\left(\mathrm{C}_{2} \mathrm{O}_{4}\right)\left(\mathrm{H}_{2} \mathrm{O}\right)_{2}\right]^{[27]}$, (b) MIL-53( $\left.\mathrm{Fe}\right)-(\mathrm{COOH})_{2}{ }^{[39]}$ 与 (c) MOF-74(Mg)-Urea- $d_{4}^{[40]}$ 的结构示意图

Figure 4 Schematic illustration of (a) $\left[\mathrm{Fe}\left(\mathrm{C}_{2} \mathrm{O}_{4}\right)\left(\mathrm{H}_{2} \mathrm{O}\right)_{2}\right]^{[27]}$, (b) MIL-53(Fe)-(COOH) $)_{2}^{[39]}$ and (c) MOF-74(Mg)-Urea- $d_{4}{ }^{[40]}$

金属-膦酸配体不仅具有酸性位点丰富、释放质子 多等优势, 还具有优异的水稳定与热稳定性, 使得其能 够应用于不同湿度/温度环境之中 ${ }^{[41]}$. Shimizu 课题组在 MOFs 质子导体领域做了很多开创性的工作, 其代表性 的贡献是合成一系列应用于不同环境中的基于金属-膦 酸配体的 PCMOF 系列化合物. 2010 年, 他们 ${ }^{[42]}$ 首先报 道了一种 $\mathrm{Zn}$-膦酸层状 MOF $\mathrm{Zn}_{3}\left(\mathrm{~L}^{3}\right)\left(\mathrm{H}_{2} \mathrm{O}\right)_{2} \cdot 2 \mathrm{H}_{2} \mathrm{O}$ (PCMOF-3). 该结构的最大特点是存在由 $\mathrm{Zn}-\mathrm{H}_{2} \mathrm{O}$ 与膦 酸基团中的氧原子组成的极化层, 可以通过强氢键作用 吸引水分子形成连续的氢键网络 (图 5a). 这使得 PCMOF-3 的活化能低至 $0.17 \mathrm{eV}$, 小于 Nafion 的数值 $(0.22 \mathrm{eV})$. 为了拓宽金属-膦酸结构的种类, 他们通过 $\mathrm{La}\left(\mathrm{HSO}_{4}\right)_{3}$ 与 $1,2,4,5$-四(磷酸亚甲基)苯 $\left(\mathrm{H}_{8} \mathrm{~L}^{9}\right)$ 配体反应
得到一种三维开放式结构 PCMOF-5 $\left(\left(\mathrm{H}_{5} \mathrm{~L}_{9}\right)\left(\mathrm{H}_{2} \mathrm{O}\right)_{3}\right.$ ・ $\left.\mathrm{H}_{2} \mathrm{O}\right)^{[43]}$. 这种 $\mathrm{MOF}$ 的孔道被膦酸基团及其配位的水分 子所占据, 形成一维链状氢键通道, 而未配位的双质子 膦酸与水分子共同组成质子供体(图 5b). 这种独特的结 构优势使得 PCMOF-5 的活化能低至 $0.15 \mathrm{eV}$. 接下来他 们 ${ }^{[44]}$ 又合成了新型 $\mathrm{Mg}$ 基 $\mathrm{MOF} \mathrm{Mg}_{2}\left(\mathrm{H}_{2} \mathrm{O}\right)_{4}\left(\mathrm{H}_{2} \mathrm{~L}^{1}\right) \cdot \mathrm{H}_{2} \mathrm{O}$ (PCMOF-10). 其每个 Mg 原子与两个水分子配位, 且每 个膦酸基团均携带一个质子, 同时充当质子供体与受 体. 配位水分子、羧酸基团与膦酸基团间形成了大范围 的氢键网络，同时层与层之间包含排列致密的结晶水， 非常适宜质子传导(图 5c). PCMOF-10 的电导率达到 $3.55 \times 10^{-2} \mathrm{~S} \bullet \mathrm{cm}^{-1}\left(70{ }^{\circ} \mathrm{C}, 95 \% \mathrm{RH}\right)$, 且能够稳定在水中 保存 $7 \mathrm{~d}$.

除了在配体修饰酸性基团外, 直接在 MOFs 孔隙内 部引入酸性分子也是一种构建导体内部氢键网络的有 效手段. 兰亚乾等 ${ }^{[45]}$ 采用水热法制备出一种新型配位 聚合物 $\left[\mathrm{Cu}_{4} \mathrm{~L}_{2}\left(\mathrm{SiW}_{12} \mathrm{O}_{40}\right)(\mathrm{OH})_{4}\left(\mathrm{H}_{2} \mathrm{O}\right)_{8}\right] \cdot 8 \mathrm{H}_{2} \mathrm{O}$. 该 $\mathrm{MOF}$ 嵌 入 $\mathrm{N}_{2} \mathrm{H}_{4} \cdot \mathrm{H}_{2} \mathrm{SO}_{4}$ 后, 质子电导率由 $1.4 \times 10^{-4} \mathrm{~S} \cdot \mathrm{cm}^{-1}$ 提升 至 $3.9 \times 10^{-3} \mathrm{~S} \cdot \mathrm{cm}^{-1}\left(65{ }^{\circ} \mathrm{C}, 98 \% \mathrm{RH}\right)$, 而活化能降至 $0.16 \mathrm{eV}$, 这主要是由于 $\mathrm{N}_{2} \mathrm{H}_{6}{ }^{+}$既能充当质子供体, 又能 作为载体携带质子在通道内移动. $\mathrm{Li}$ 等 ${ }^{[46]}$ 成功将 $\mathrm{H}_{2} \mathrm{SO}_{4}$ 分子嵌入 $\mathrm{MIL}-101-\mathrm{SO}_{3} \mathrm{H}$ 的孔隙中，得到 $\mathrm{H}_{2} \mathrm{SO}_{4} @ \mathrm{MIL}-101-\mathrm{SO}_{3} \mathrm{H}$ 复合结构. 该结构的质子电导率 达到了惊人的 $1.82 \mathrm{~S} \cdot \mathrm{cm}^{-1}\left(70{ }^{\circ} \mathrm{C}, 90 \% \mathrm{RH}\right)$, 且在 $-40{ }^{\circ} \mathrm{C}$ 的低温下仍有 $0.92 \times 10^{-2} \mathrm{~S} \cdot \mathrm{cm}^{-1}$, 是有报道的 MOFs 质子导体在低温下的最高值, 这主要是由于该 $\mathrm{MOF}$ 内部双质子源 $\left(\mathrm{H}_{2} \mathrm{SO}_{4}\right.$ 与 $\left.-\mathrm{SO}_{3} \mathrm{H}\right)$ 的协同作用.

MOFs 晶体中的结构转变与单晶-单晶转变对氢键 网络的形成与排列起到关键作用, 进而影响质子电导 率. 李建荣等 ${ }^{[28]}$ 报道了一种具有柔性结构的 MOF BUT- $8(\mathrm{Cr})$ A, 其三维结构由无数富集磺酸基位点的一 维通道构成. 高湿度条件下 BUT-8(Cr)A 内部金属离子 簇与菜环可沿磺酸基团 $\mathrm{O}-\mathrm{O}$ 键转动, 保证了
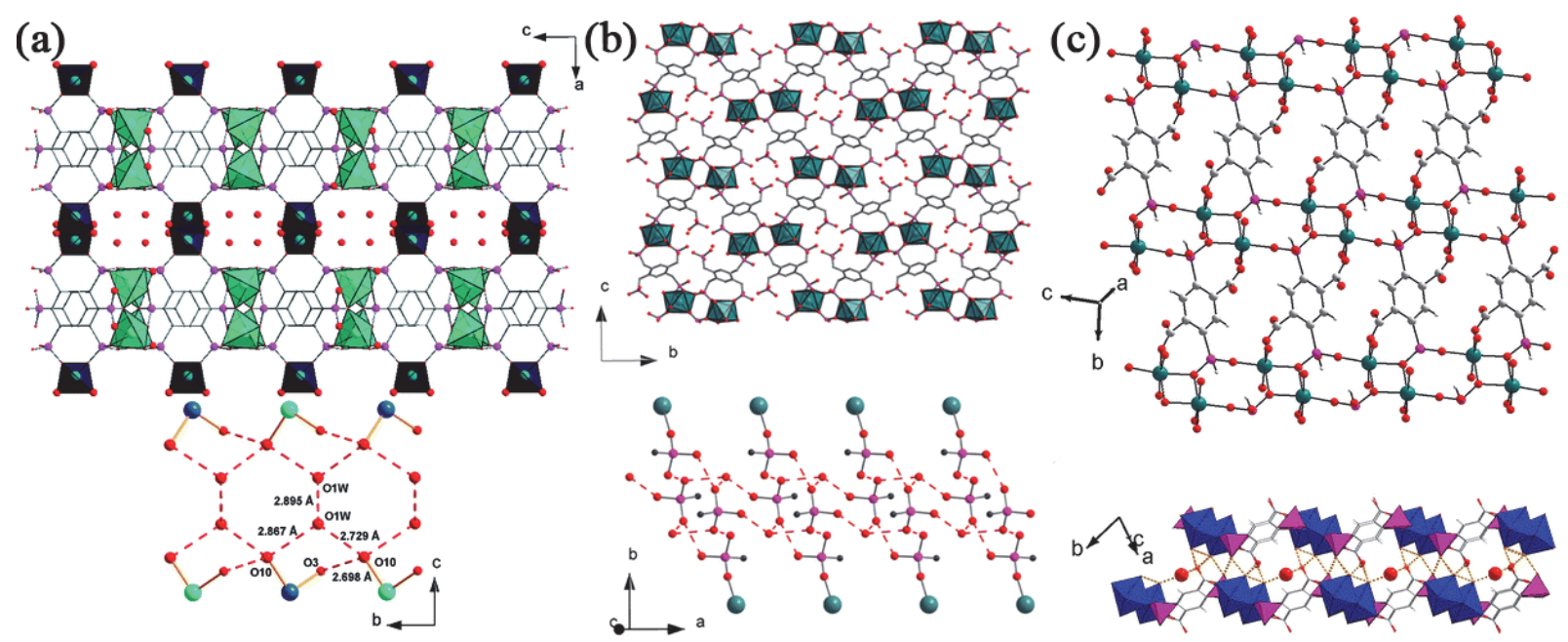

图 5 (a) PCMOF-3 $3^{[42]}$, (b) PCMOF-5 $5^{[43]}$ 与(c) PCMOF-10 $0^{[44]}$ 的结构示意图(上)与质子传递通道示意图(下)

Figure 5 Schematic illustration of structures (top) and proton conducting channel (bottom) of (a) PCMOF- $3^{[42]}$, (b) PCMOF-5 $5^{[43]}$ and (c) PCMOF-10 $0^{[44]}$ 
BUT-8(Cr)A 结构对湿度的自适应性; 而未引入磺酸基 才时 MOF 骨架呈刚性, 在低湿度条件下无法收缩保持 氢键网络(图 6a). BUT- $8(\mathrm{Cr}) \mathrm{A}$ 在 $80{ }^{\circ} \mathrm{C}, 100 \% \mathrm{RH}$ 的条件 下质子电导率达到 $1.27 \times 10^{-1} \mathrm{~S} \cdot \mathrm{cm}^{-1}$, 且在低湿度条件 下仍能保持约 $10^{-3} \mathrm{~S} \cdot \mathrm{cm}^{-1}$ 的水平. $\mathrm{Li}$ 等 ${ }^{[47]}$ 报道了一种 特殊的二维配位聚合物 $\left[\mathrm{Cu}_{2}(\mathrm{Htzehp})_{2}\left(4,4{ }^{\prime}-\right.\right.$-bipy $\left.)\right] \cdot 3 \mathrm{H}_{2} \mathrm{O}$ $\left(1 \cdot 3 \mathrm{H}_{2} \mathrm{O}\right)$, 其在氮气气氛下于 $80{ }^{\circ} \mathrm{C}$ 会因结晶水蒸发产 生可逆单晶-单晶转变(图 $6 \mathrm{~b}$ ), 导致化合物 $b$ 轴与 $c$ 轴长 度缩短, 证明该方向水分子搭建的氢键通道的存在. 其 单晶在 $80{ }^{\circ} \mathrm{C}, 95 \% \mathrm{RH}$ 下沿[100]面的质子电导率为 1.43 $\times 10^{-3} \mathrm{~S} \cdot \mathrm{cm}^{-1}$, 在 $48 \mathrm{~h}$ 之内保持稳定, 且较 [010]面高约 2 个数量级(图 $6 \mathrm{c}$ ).

近年来, 有水条件下工作的 MOFs 质子导体成果丰 硕, 且一些 MOFs 的质子电导率甚至超越了 Nafion $\left(>10^{-2} \mathrm{~S} \cdot \mathrm{cm}^{-1}\right)$, 但是大部分最优电导率都是在最大湿 度所得, 且数值随湿度变化波动很大, 有的甚至在几个 数量级. 因此, 如何保持 MOFs 质子导体在宽湿度范围 内的质子传输稳定性及考察在实际质子交换膜燃料电 池系统中的表现是需要关注的重点.

\section{2 无水条件下工作的 MOFs 质子导体}

迄今为止, 很多 MOFs 的质子导电过程都需要在 $100{ }^{\circ} \mathrm{C}$ 以下的高湿度环境进行. 然而为了提高燃料电池 的反应效率, 并且拓宽燃料电池的应用范围, 探求在高 温、无水环境下应用的质子导体至关重要. 近年来, 研 究者们通过选用合适的中心金属及配体, 合成了可在 $100{ }^{\circ} \mathrm{C}$ 以上高温进行应用的 MOFs 质子导体. 例如 Kitagawa 等 ${ }^{[19]}$ 使用膦酸盐为配体合成了 $\left[\mathrm{Zn}\left(\mathrm{H}_{2} \mathrm{PO}_{4}\right)_{2}(\mathrm{TzH})_{2}\right]_{n}$ 结构, 其在 $150{ }^{\circ} \mathrm{C}$ 的无水环境下质 子电导率为 $1.2 \times 10^{-4} \mathrm{~S} \cdot \mathrm{cm}^{-1}$. Nagarkar 等 ${ }^{[48]}$ 合成了一
种特殊的 $\mathrm{Zn}-\mathrm{MOF}\left\{\left[\left(\mathrm{Me}_{2} \mathrm{NH}_{2}\right)_{3}\left(\mathrm{SO}_{4}\right)\right]_{2}\left[\mathrm{Zn}_{2}(\mathrm{ox})_{3}\right]\right\}_{n}$. 这种 MOF 在无水环境下 $\left(1 \times 10^{-4} \mathrm{~S} \cdot \mathrm{cm}^{-1}, 150{ }^{\circ} \mathrm{C}\right)$ 与高湿度 环境下 $\left(4.2 \times 10^{-2} \mathrm{~S} \cdot \mathrm{cm}^{-1} 98 \% \mathrm{RH}\right)$ 均可完成质子导电.

更加常用的提升 MOFs 高温质子导电性的方法是在 其孔道内部嵌入客体分子，以代替水分子完成氢键网络 的搭建和质子传递过程. 咪唑(Imidazone, Im)、三唑类含 氮杂环物质具有低挥发性和高沸点等特性, 且当温度达 到结晶熔点(均为 $80{ }^{\circ} \mathrm{C}$ 左右)时有极佳的质子导电性. 基于上述特性，咪唑及三唑类分子被广泛用做高温、无 水环境下 MOFs 质子导体的客体分子. Shimizu 课题 组 ${ }^{[49]}$ 在 2009 年报道了一种 $\beta$-PCMOF2 结构, 第一次向 世人展现了 MOFs 质子导体在高温环境的应用潜力. $\beta$-PCMOF2 三维结构内存在直径约为 $0.5 \mathrm{~nm}$ 的一维孔 道(图 7a). 当该孔道内部填充了 $1 H-1,2,4-$ 三氮唑后, $\beta$-PCMOF2 的在 $150{ }^{\circ} \mathrm{C}$ 下的质子电导率达到了 $5 \times 10^{-4}$ $\mathrm{S} \cdot \mathrm{cm}^{-1}$. 同年, Kitagawa 课题组 ${ }^{[50]}$ 合成了孔道负载了咪 唑的两种含铝 MOFs 结构, $\mathrm{Al}\left(\mu_{2}-\mathrm{OH}\right)(1,4-\mathrm{ndc})$ 与 $\mathrm{Al}\left(\mu_{2}-\mathrm{OH}\right)(1,4-\mathrm{bdc})$. 二者的室温质子电导率分别约为 $10^{-8}$ 与 $10^{-10} \mathrm{~S} \cdot \mathrm{cm}^{-1}$; 而当温度升高至 $120{ }^{\circ} \mathrm{C}$ 时, 其质 子电导率提升了三个数量级. 两者间质子电导率的差异 主要是咪唑与孔道内部基团的作用力不同(图 7b 与 7c), 使得客体分子在 MOFs 结构内部运动能力产生差异. 2014 年, Kitagawa 组 ${ }^{[33]}$ 制备了一种含铜 $\mathrm{MOF}$ $\left[\mathrm{ImH}_{2}\right]\left[\mathrm{Cu}\left(\mathrm{H}_{2} \mathrm{PO}_{4}\right)_{2} \mathrm{Cl}\right] \cdot \mathrm{H}_{2} \mathrm{O}$, 其晶体结构在 $70{ }^{\circ} \mathrm{C}$ 时会从 有序转为无序, 产生大量可移动的 Im 质子载体, 使得 其无水条件下的质子电导率高达 $2 \times 10^{-2} \mathrm{~S} \cdot \mathrm{cm}^{-1}$ $\left(130{ }^{\circ} \mathrm{C}\right)$. 这项工作成功将 MOFs 的无水电导率提高到 与 Nafion 相当的水平. 近年来, Ye 等 ${ }^{[51]}$ 与 Zhang 等 ${ }^{[32]}$ 开展的研究细致探讨了咪唑的存在形式(配体或游离态) 及排列方式对 MOF 质子导体性能的影响.
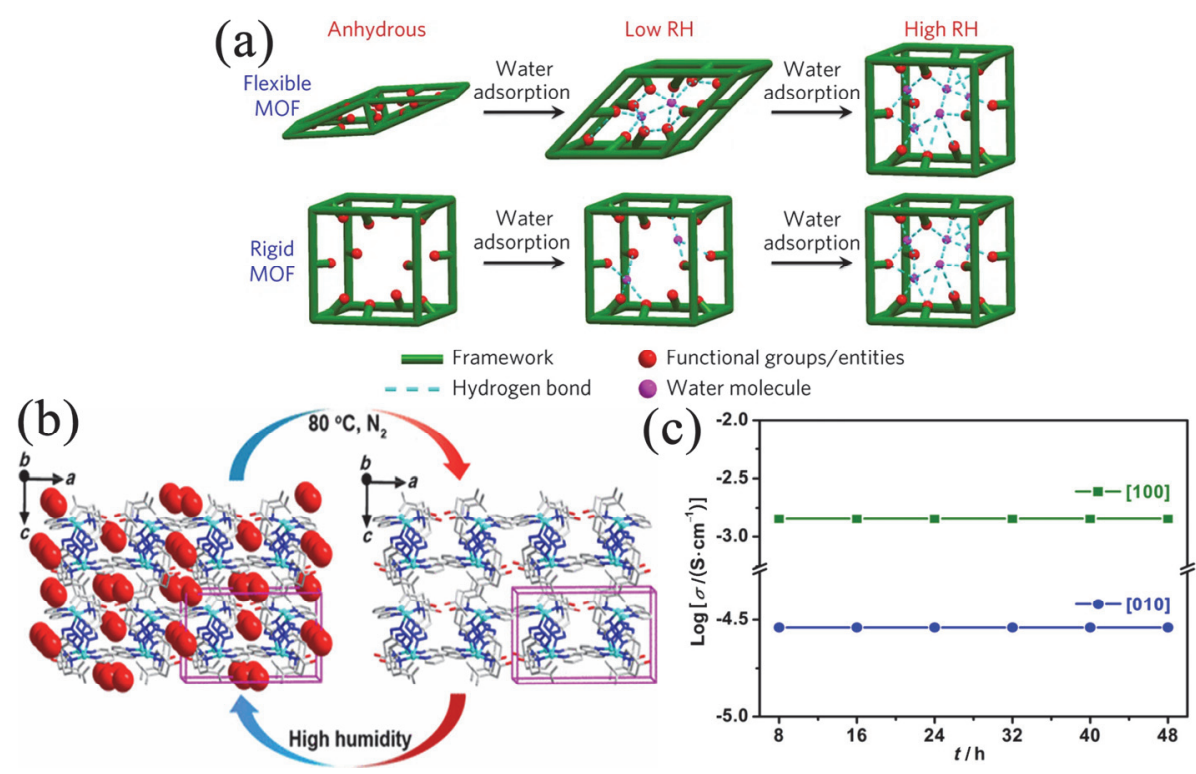

图 6 (a) BUT- $8(\mathrm{Cr}) \mathrm{A}$ 的结构随湿度变化机理 ${ }^{[28]}$, (b) $1 \cdot 3 \mathrm{H}_{2} \mathrm{O}$ 的单晶转变示意图与(c) $1 \cdot 3 \mathrm{H}_{2} \mathrm{O}$ 在[010]与 $[010]$ 面方向的质子电导率 $\left(80{ }^{\circ} \mathrm{C}, 95 \% \mathrm{RH}\right)^{[47]}$ Figure 6 Schematic illustration of framework variation for (a) BUT-8(Cr)A under anhydrous, low RH and high $\mathrm{RH}^{[28]}$ and (b) single-crystsal transformation of $\mathbf{1} \cdot 3 \mathrm{H}_{2} \mathrm{O}$. (c) Time-dependent proton conductivity of the single crystals of $\mathbf{1} \cdot 3 \mathrm{H}_{2} \mathrm{O}$, as measured along the [100] and [010] directions at $80{ }^{\circ} \mathrm{C}$ and $95 \% \mathrm{RH}^{[47]}$. 

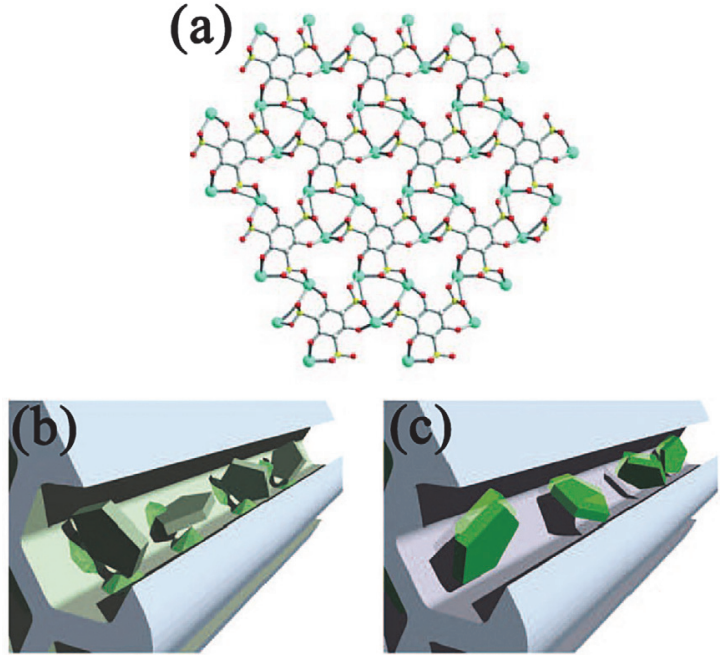

图 7 (a)单层 $\beta$-PCMOF2 的球棍模型示意图, 显示出由 18 圆环组成 的孔道. (b)孔道内发生强配位作用时, 会限制咪唑移动, 质子电导率 低下. (c)纳米孔道内的咪唑以弱配位力协同, 因此分子可以自由移动, 产生高质子电导率 ${ }^{[50]}$

Figure 7 (a) A ball-and-stick representation of a single layer showing the open 18-membered channels of $\beta$-PCMOF2 $2^{[49]}$. (b) The strong host-guest interaction retards the mobility of imidazole to afford the low proton conductivity. (c) Imidazoles are accommodated in a nanochannel without strong host-guest interaction, and therefore, the molecules obtain the high mobility to show high proton conductivity ${ }^{[50]}$

离子液体(Ionic Liquid, IL)是一种安全、使用温度适 中、高热稳定性、无挥发性、不可燃、腐蚀性低的电解 液. 将离子液体嵌入 MOFs 结构扩展了 MOFs 质子导体 在中高温条件下的应用潜力. Sun 等 ${ }^{[2]}$ 将一种二元离子 液体 EIMS-HTFSA 成功嵌入 MIL-101 的通道内, 形成 EIMS-HTFSA@MIL 结构. 离子液体的引入使得 MIL-101 显示出稳定的高温质子传递能力 $\left(2 \times 10^{-4} \mathrm{~S}\right.$ ・ $\left.\mathrm{cm}^{-1}, 140{ }^{\circ} \mathrm{C}\right)$. Chen 等 ${ }^{[36]}$ 考察了三种不同离子液体
SA-EIMS, MSA-EIMS 与 PTSA-EIMS 嵌入 MIL-101 后 (IL@MIL-101) 质子传递能力的变化. 结果显示三种 IL@MIL-101 均表现出可观的质子电导率和 ILs 的长期 稳定性, 其中 SA-EIMS@MIL-101 达到最高值 1.89× $10^{-3} \mathrm{~S} \cdot \mathrm{cm}^{-1}\left(150{ }^{\circ} \mathrm{C}\right)$. 三种产物的质子导电能力的差异 来源于 IL@MIL-101 内阴离子 $\mathrm{R}^{-\mathrm{SO}_{3}}{ }^{-}$的差异: 阴离子体 积越大, 越阻碍质子通道, 使得传导能力降低.

目前, 无水 MOFs 质子导体研究尚处于起步阶段, 制备在低温及中高温均能保持较高电导率的 MOFs 仍存 在较大挑战. 此外, 对于无客体分子的无水 MOFs 质子 导体研究不仅能拓宽无水质子导体的种类, 还可进一步 认识 MOFs 结构与质子导电间的构效关系, 因此需要在 未来的工作中更加重视.

\section{MOFs 质子交换膜}

目前为止, 大部分关于 MOFs 质子导体的研究都是 基于粉末压片或单晶进行的. 然而, 要想真正将 MOFs 质子导体用在燃料电池系统, 关键是将粉末或单晶样品 转化成膜结构. 目前已报道的 MOFs 质子交换膜包含纯 MOFs 质子导体薄膜, 和以 MOFs 质子导体作为分散相 的混合基质膜(mixed matrix membranes, MMMs). 表 2 总结了近年来一些代表性的基于 MOFs 的质子交换膜的 关键性能.

\subsection{MOFs 薄膜结构质子交换膜}

最早关于 MOFs 薄膜的制备报道可见于 2005 年 ${ }^{[33]}$, 然而直到 2013 年 ${ }^{[54]}$ 才有关于 MOFs 薄膜用做质子导体 的相关研究. 这是因为应用于质子交换领域的 MOFs 薄 膜需要规整、致密、连续、零缺陷 ${ }^{[55,56]}$, 而制备具有以 上特征的 MOFs 薄膜一直是一大挑战.

表 2 一些已报道的 MOF 质子交换膜的关键性能

Table 2 Key properties of some reported MOF-based proton exchange membranes

\begin{tabular}{|c|c|c|c|c|c|c|}
\hline MOF-based membranes & Structures & $\begin{array}{l}\text { Conductivity/ } \\
\left(\mathrm{S} \cdot \mathrm{cm}^{-1}\right)\end{array}$ & $\begin{array}{l}\text { Methanol per- } \\
\text { meability/ } \\
\left(\mathrm{cm}^{2} \cdot \mathrm{s}^{-1}\right)\end{array}$ & Work condition & $E_{\mathrm{a}} / \mathrm{eV}$ & Ref. \\
\hline Cu-TMA/SPEEK & Cu-TMA MOF in SPEEK matrix & $4.5 \times 10^{-2}$ & $4.26 \times 10^{-7}$ & $70{ }^{\circ} \mathrm{C}, 98 \% \mathrm{RH}$ & 0.075 & {$[57]$} \\
\hline DNA@ZIF-8 & DNA-threaded ZIF-8 thin film & $1.7 \times 10^{-1}$ & $1.25 \times 10^{-8}$ & $75{ }^{\circ} \mathrm{C}, 97 \% \mathrm{RH}$ & 0.44 & [58] \\
\hline PSS@ZIF-8 & $\begin{array}{l}\text { Poly(4-styrenesulfonate)-encapsulated ZIF-8 thin } \\
\text { film }\end{array}$ & $2.59 \times 10^{-1}$ & $2.08 \times 10^{-9}$ & $80{ }^{\circ} \mathrm{C}, 100 \% \mathrm{RH}$ & 0.543 & [59] \\
\hline Nafion-Cage 3 & Crystallized Cage 3 in Nafion matrix & $2.7 \times 10^{-1}$ & $5.82 \times 10^{-8}$ & $90{ }^{\circ} \mathrm{C}, 95 \% \mathrm{RH}$ & $\mathrm{N} / \mathrm{A}^{a}$ & {$[60]$} \\
\hline GO@UiO-66- $\mathrm{NH}_{2} /$ Nafion & $\begin{array}{l}\mathrm{UiO}-66-\mathrm{NH}_{2} \text { was tethered onto graphene oxide and } \\
\text { then into Nafion matrix }\end{array}$ & $\begin{array}{l}3.03 \times 10^{-1} \\
3.403 \times 10^{-3}\end{array}$ & $1.74 \times 10^{-8}$ & $\begin{array}{l}90{ }^{\circ} \mathrm{C}, 95 \% \mathrm{RH} \\
120{ }^{\circ} \mathrm{C} \text {, anhydrous }\end{array}$ & $\mathrm{N} / \mathrm{A}^{a}$ & {$[62]$} \\
\hline Ni-BDC/PAN@imidazole & $\begin{array}{l}\text { Imidazole coupled Ni-BDC nanosheets-PAN nano- } \\
\text { fibers membrane }\end{array}$ & $6.04 \times 10^{-5}$ & $\mathrm{~N} / \mathrm{A}^{a}$ & $90{ }^{\circ} \mathrm{C}, 90 \% \mathrm{RH}$ & 0.819 & {$[63]$} \\
\hline $\mathrm{H}_{3} \mathrm{PO}_{4} @ \mathrm{Ni}-\mathrm{BDC}-\mathrm{PAN}$ & $\mathrm{H}_{3} \mathrm{PO}_{4}$-encapsulated Ni-BDC-PAN membrane & $1.05 \times 10^{-2}$ & $\mathrm{~N} / \mathrm{A}^{a}$ & $80{ }^{\circ} \mathrm{C}, 90 \% \mathrm{RH}$ & 0.82 & [64] \\
\hline ZIF-8@GO/Nafion & In-situ growth ZIF-8 on GO doped in Nafion matrix & $2.8 \times 10^{-1}$ & ca. $3 \times 10^{-7}$ & $120{ }^{\circ} \mathrm{C}, 40 \% \mathrm{RH}$ & 0.137 & [65] \\
\hline IL@ $\mathrm{NH}_{2}$-MIL-101/SPAEK & $\begin{array}{l}\text { Ionic liquid-impregnated } \mathrm{NH}_{2} \text {-MIL-101 into SPAEK } \\
\text { matrix }\end{array}$ & $1.84 \times 10^{-1}$ & $7.53 \times 10^{-7}$ & $\begin{array}{l}80{ }^{\circ} \mathrm{C} \text {, deionized } \\
\text { water }\end{array}$ & 0.139 & {$[66]$} \\
\hline $\begin{array}{l}\mathrm{UiO}-66-\mathrm{NH}_{2}+ \\
\text { UiO-66- } \mathrm{SO}_{3} \mathrm{H} / \text { Nafion }\end{array}$ & UiO-66- $\mathrm{NH}_{2}+\mathrm{UiO}-66-\mathrm{SO}_{3} \mathrm{H}$ codopped into Nafion & $2.56 \times 10^{-1}$ & $4.41 \times 10^{-8}$ & $90{ }^{\circ} \mathrm{C}, 95 \% \mathrm{RH}$ & $\mathrm{N} / \mathrm{A}^{a}$ & [68] \\
\hline $\mathrm{CS} / \mathrm{A}+\mathrm{B}$ & $\begin{array}{l}\mathrm{UiO}-66\left(\mathrm{SO}_{3} \mathrm{H}\right)(\mathbf{A})+\mathrm{UiO}-66\left(\mathrm{NH}_{2}\right)(\mathbf{B}) \text { into Chitosan } \\
\text { matrix }\end{array}$ & $\begin{array}{l}3.78 \times 10^{-3} \\
5.2 \times 10^{-2}\end{array}$ & $\mathrm{~N} / \mathrm{A}^{a}$ & $\begin{array}{l}120{ }^{\circ} \mathrm{C} \text {, anhydrous } \\
100{ }^{\circ} \mathrm{C}, 98 \% \mathrm{RH}\end{array}$ & 0.131 & [69] \\
\hline
\end{tabular}

${ }^{a} \mathrm{~N} / \mathrm{A}$ : 文中未提及(Not available). 
Kitagawa 等 ${ }^{[54]}$ 制备了一种具有高度取向性和结晶 性的 $\mathrm{Cu}-\mathrm{TCPP}$ MOF 薄膜. $\mathrm{Cu}-\mathrm{TCPP}$ 是一种二维纳米片 结构 MOF, 其配体在 $a b$ 面通过 $\mathrm{TCPP}$ (四(4-羧基苯基) 卟啉)配体相互连接, 并在 $c$ 轴以 $\mathrm{AB}$ 型方式完成堆叠. 他们首先用溶剂热法得到了 $\mathrm{Cu}-\mathrm{TCPP}$ 纳米片, 而后通 过分子自组装法将纳米片悬浮液沉积在 $\mathrm{SiO}_{2} / \mathrm{Si}$ 基底上, 最终形成 Cu-TCPP 薄膜(图 8a). 掠入射 $\mathrm{X}$ 射线衍射 (GIXRD)分析结果(图 $8 \mathrm{~b}$ 与 $8 \mathrm{c}$ )表明 $\mathrm{Cu}-\mathrm{TCPP}$ 纳米片的 $a b$ 面平行于薄膜基底, 使其表现出高度的取向性. 在 $25{ }^{\circ} \mathrm{C}$ 时, 随相对湿度的增加, $\mathrm{Cu}-\mathrm{TCPP}$ 薄膜的电导率由 $3.2 \times 10^{-8} \mathrm{~S} \cdot \mathrm{cm}^{-1}(40 \% \mathrm{RH})$ 猛增至 $3.9 \times 10^{-3} \mathrm{~S} \cdot \mathrm{cm}^{-1}$ $(98 \% \mathrm{RH})$. 这是首个将 MOFs 薄膜应用于质子交换领域 的报道.
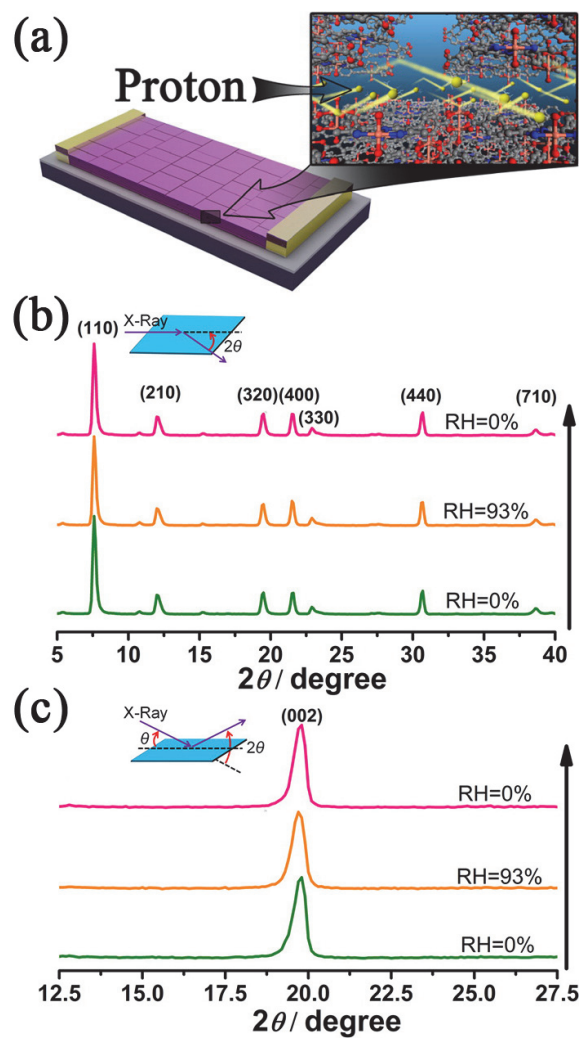

图 $8 \mathrm{Cu}$-TCPP 薄膜(a)在 $\mathrm{SiO}_{2} / \mathrm{Si}$ 基底与 $\mathrm{Au} / \mathrm{Cr}$ 电极上的示意图及(b) 沿 $a b$ 面与(c)垂直 $a b$ 面的 GIXRD 谱图 ${ }^{[54]}$

Figure 8 (a) Schematic illustration of Cu-TCPP film on $\mathrm{SiO}_{2} / \mathrm{Si}$ substrate and $\mathrm{Au} / \mathrm{Cr}$ electrode. GIXRD pattern of Cu-TCPP film: (b) in $a b$ plane and (c) out of $a b$ plane ${ }^{[54]}$

类似地, Zhang 等 ${ }^{[67]}$ 使用电化学法在铜片基底上合 成了多孔 NENU-3 薄膜. 他们选用磷铇酸(PTA)作为合 成电解液, 同时 PTA 会渗入 NENU-3 的孔隙内成为质子 传递的客体分子. 该薄膜在室温至 $280{ }^{\circ} \mathrm{C}$ 范围内保持 高度的热稳定性、水稳定性与抗氧化性. 其在 $24{ }^{\circ} \mathrm{C}$, $98 \% \mathrm{RH}$ 的电导率数值为 $1.4 \times 10^{-5} \mathrm{~S} \cdot \mathrm{cm}^{-1}$, 对应活化能 为 $0.38 \mathrm{eV}$, 符合 Grotthuss 机理.

受生物大分子内部离子通道的启发, 蒋仲杰、陈邦 林与彭新生等 ${ }^{[58]}$ 合作采用原位生长法制备了孔隙贯穿 DNA 分子的 ZIF-8 膜结构(DNA@ZIF-8). DNA 分子的加
入使得复合膜的质子导电率最高可以达到 $0.17 \mathrm{~S} \cdot \mathrm{cm}^{-1}$ $\left(75{ }^{\circ} \mathrm{C}, 97 \%\right.$ RH). DNA@ZIF-8 结构内部水分子与 DNA 分子共同组成的长程质子传递通道, 使得质子以 Grotthuss 机制进行运输(图9). 该薄膜结构致密, 热稳定 性强, 且甲醇渗透率仅为 $1.25 \times 10^{-8} \mathrm{~cm}^{2} \cdot \mathrm{s}^{-1}$, 最大输出 功率密度达到 $9.87 \mathrm{~mW} \cdot \mathrm{cm}^{-2}$, 使得其在燃料电池环境 应用具有巨大潜力. Cai 等 ${ }^{[59]}$ 采用同样方式制备了聚苯 乙烯磺酸(PSS)链交联的 ZIF-8 复合薄膜. 当 PSS 的质量 分数为 $9 \%$ 时, 其质子电导率达到 $2.59 \times 10^{-1} \mathrm{~S} \cdot \mathrm{cm}^{-1}$ $\left(80{ }^{\circ} \mathrm{C}, 100 \% \mathrm{RH}\right)$. 该复合膜的致密结构使其在水中不 发生溶胀现象，且甲醇渗透率仅为 $2.08 \times 10^{-9} \mathrm{~cm}^{2} \bullet \mathrm{s}^{-1}$, 较 Nafion 低三个数量级.
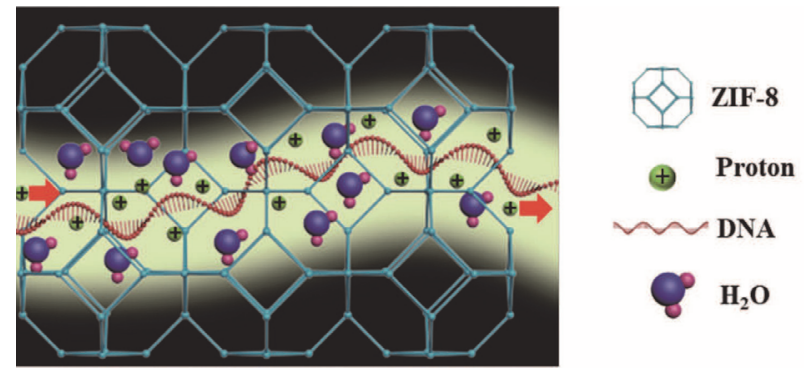

图9 DNA@ZIF-8 的质子传递机理示意图 ${ }^{[58]}$

Figure 9 Illustration of the proton transportation mechanism of DNA@ZIF-8 membrane ${ }^{[58]}$

\subsection{MOFs 混合基质质子交换膜}

混合基质膜是指无机或无机/有机杂化分散相与聚 合物基体共同组成的膜结构. 这种新型膜结构有效结合 了无机材料与有机材料的优势，同时克服了各自的缺 点, 如无机材料的难成膜性质与高分子材料性能的长程 无序性. 近年来，以 MOFs 为增强相的混合基质膜发展 迅速，并在气体吸附与分离等领域展现出巨大应用潜 力 $^{[70-72]}$. 然而, 尽管 MOFs 质子导体的发展迅猛, MOFs 复合质子交换膜的报道却相对较少. 这主要是因以下问 题导致: (1) MOFs 与高分子基体的界面结合不好, 会形 成缺陷破坏质子传输通道; (2)一般采用粉末相 MOFs 制 备混合基质膜，填充相的质量分数很难超过 $10 \%$, 否则 即会发生颗粒聚集现象，造成电导率下降; (3)加入增强 相使复合膜质子交换性能提高的同时，不可避免会带来 燃料渗透率的损失，一定程度上影响电池环境的实际使 用 ${ }^{[73-78]}$. 基于以上问题，研究者们尝试了多种途径以提 高基于 MOFs 的混合基质质子交换膜的性能.

\subsubsection{MOFs 粉体作为增强相的混合基质质子交换膜}

为提高 MOFs 粉体在基体内的分散程度, 需要对 MOFs 表面进行修饰. $\mathrm{Ru}$ 等 ${ }^{[79]}$ 向磺化聚芳醚酮 (SNF-PAEK) 基体内加入了修饰后的 MIL-101 (MNS). 当 $\mathrm{MNS}$ 的加入量为 $3 \%$ (质量分数)时, MNS@SNFPAEK 复合膜的热稳定性与抗氧化性大幅提高, 且拉伸 强度(35.47 $\mathrm{MPa})$ 与模量 $(0.97 \mathrm{GPa})$ 都显著高于 Nafion. MNS 的加入使膜的电导率达到 $0.192 \mathrm{~S} \cdot \mathrm{cm}^{-1}$, 且选择性 
较纯 SNF-PAEK 提升了一倍, 同时输出功率密度达到 $125.7 \mathrm{~mW} \cdot \mathrm{cm}^{-2}$, 展现出燃料电池环境下的巨大应用潜 力. 小角度 $\mathrm{X}$ 射线衍射 (SAXS)与 TEM 分析证明了对 MIL-101 的修饰使得 MNS 在基体周围形成了明显的亲 水层, 在 $\mathrm{MNS}$ 与基体间构筑了质子传递路径(图 10), 提 高了质子导电性.
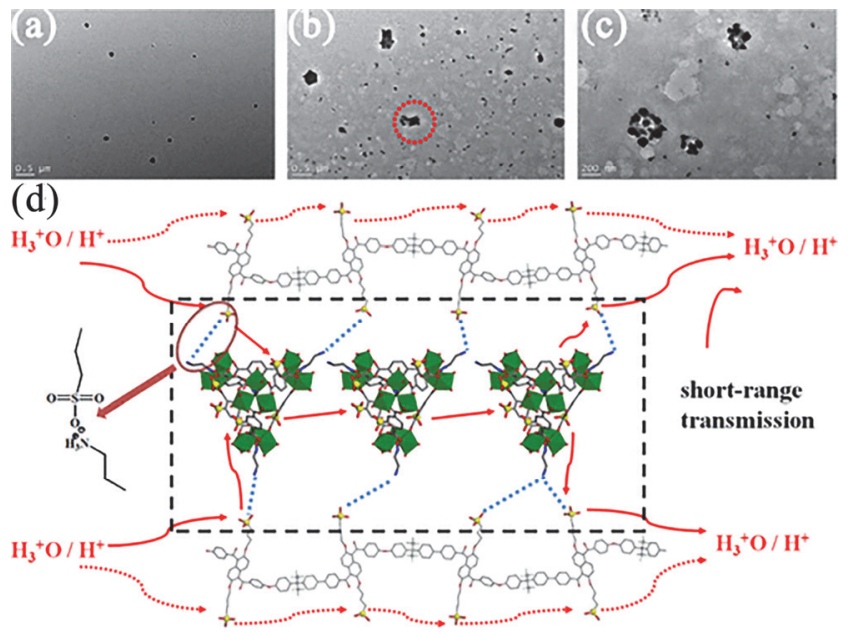

图 10 (a) (c) MNS@SNF-PAEK 经 $\mathrm{AgNO}_{3}$ 溶液染色后的 TEM 图像. (d) MNS@SNF-PAEK 可能的质子传递路径示意图 ${ }^{[79]}$

Figure 10 (a) (c) TEM images of MNS@SNF-PAEK after $\mathrm{AgNO}_{3}$ dying. (d) Schematic illustration of proton conducting channels of MNS@SNF-PAEK
UiO-66 制备成本低，合成简单，同时也具有良好的 质子传递能力，是复合质子交换膜增强相的合适选 择 ${ }^{[80,81]}$. Liu 等 ${ }^{[82]}$ 研究了含不同基团的 UiO-66 对磺化聚 醚醚酮(SPEEK)膜性能的影响. 他们通过改变苯二甲酸 配体的种类, 得到了纳米级 UiO-66, UiO-66-OH 与 UiO-66- $\mathrm{NH}_{2}$ 三种结构. 分别向 SPEEK 基体中掺入质量 分数 $0.5 \%$ 的三种 $\mathrm{UiO}-66$ 后, 膜的选择性显著提高, 特 别是 $\mathrm{S} / \mathrm{UiO}-66-\mathrm{OH}$ 的选择性达到 $12.30 \times 10^{4} \mathrm{~S} \cdot \mathrm{min} \cdot$ $\mathrm{cm}^{-3}$, 是商用 N115 隔膜的 3 倍和纯 SPEEK 隔膜的 2 倍, 这是由于-OH 基团同 Zr-MOF 协同起到在界面处防止电 解液污染的作用.

复旦大学武培怡组 ${ }^{[68]}$ 尝试将 $\mathrm{UiO}-66-\mathrm{SO}_{3} \mathrm{H}$ 与 UiO-66- $\mathrm{NH}_{2}$ 共同掺入 Nafion 基体后, 发现共混复合膜 的质子电导率 $\left(0.256 \mathrm{~S} \cdot \mathrm{cm}^{-1}\right)$ 较 Nafion、 UiO-66- $-\mathrm{SO}_{3} \mathrm{H} /$ $\mathrm{Nafion}$ 与 UiO-66- $\mathrm{NH}_{2} / \mathrm{Nafion}$ 都要高(图 11a, 11b 与 11d). $-\mathrm{SO}_{3} \mathrm{H}$ 与- $\mathrm{NH}_{2}$ 的协同作用使 UiO-66/Nafion 复合膜形成 更连续的亲水区域，构建了大范围的氢键网络. 同时他 们发现 UiO-66 的粒径越小, 复合膜的质子电导率提升 越明显(图 11c), 这是由于小粒径 MOF 和 Nafion 基体的 配合度更高.

同样, 对 MOFs 的孔道进行修饰也可增强其与复合 膜基体的协同作用. Zhang 等 ${ }^{[83]}$ 合成了一种孔隙富集大 量磷铇酸的 MIL-101(Cr)结构(HPW@MIL101), 并用铸
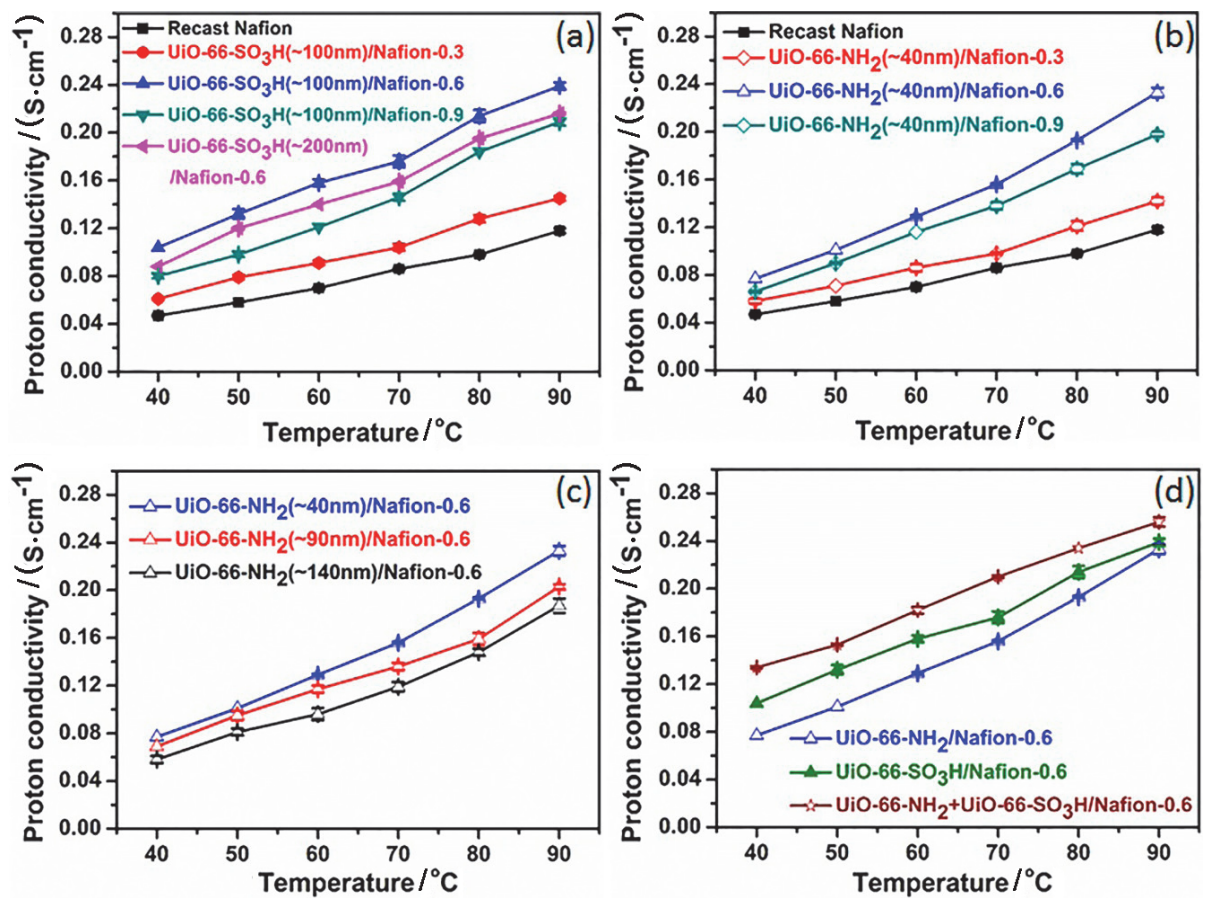

图 11 温度-质子电导率关系图: (a)铸膜法制备的 Nafion, UiO-66- $\mathrm{SO}_{3} \mathrm{H}$ (尺寸为约 100/约 $200 \mathrm{~nm}$ )/ Nafion; (b) 铸膜法制备的 Nafion 与 UiO-66- $\mathrm{NH}_{2}$ (尺寸为约 $40 \mathrm{~nm}$ )/Nafion ${ }^{[68]}$; (c) 不同尺寸 UiO-66- $\mathrm{NH}_{2} / \mathrm{Nafion}-0.6$; (d) UiO-66- $\mathrm{NH}_{2} / \mathrm{Nafion}-0.6, \mathrm{UiO}-66-\mathrm{SO}_{3} \mathrm{H} / \mathrm{Nafion}-0.6$ 与 UiO-66-NH 2 $+\mathrm{UiO}-66-\mathrm{SO}_{3} \mathrm{H} / \mathrm{Nafion}-0.6$

Figure 11 Temperature-dependent proton conductivities (95\% RH): (a) the recast Nafion, UiO-66- $\mathrm{SO}_{3} \mathrm{H}(c a .100$ and $c a$. $200 \mathrm{~nm}) / \mathrm{Nafion}$; (b) the recast Nafion, UiO-66- $\mathrm{NH}_{2}$ (ca. $\left.40 \mathrm{~nm}\right) / \mathrm{Nafion}$; (c) UiO-66- $\mathrm{NH}_{2}$ (ca. 40, $c a .90$, and $\left.c a .140 \mathrm{~nm}\right) / \mathrm{Nafion}-0.6$; and (d) UiO-66-NH $-\mathrm{Nafion}-0.6$, $\mathrm{UiO}-66-\mathrm{SO}_{3} \mathrm{H} / \mathrm{Nafion}-0.6$, and UiO-66- $\mathrm{NH}_{2}+\mathrm{UiO}-66-\mathrm{SO}_{3} \mathrm{H} / \mathrm{Nafion}-0.6^{[68]}$ 
膜法制备了 SPEEK/HPW@MIL 复合质子交换膜. MOFs 中不饱和配位的金属离子解离水产生的羟基与磷铇酸 共同作用, 使 SPEEK 与 HPW@MIL-101 界面处形成了 新的纳米质子传递通道. 其质子电导率 $\left(0.272 \mathrm{~S} \cdot \mathrm{cm}^{-1}\right.$, $100 \% \mathrm{RH}$ )较 SPEEK/MIL-101 提高了约 45.5\%; 同时在 低湿度下 $(40 \% \mathrm{RH})$ 的质子电导率是 SPEEK/MIL101 的 7.25 倍. $\mathrm{Ru}$ 等 ${ }^{[66]}$ 通过向 $\mathrm{NH}_{2}$-MIL-101 中浸渍离子液体 (IL@MOF-1), 使之与 SPAEK 基体通过氨基产生交联作 用, 大幅提高复合膜 IL@MOF-1 的形稳性、强度与抗氧 化性, 且这种交联将复合膜的燃料渗透率大幅缩减至 $6.77 \times 10^{-7} \mathrm{~cm}^{2} \cdot \mathrm{s}^{-1}$.

MOFs 与基体之间缺少一定的化学键作用也是影响 质子交换膜性能的因素, 这是因为一般的 MMMs 制备 过程中填充相与基体仅仅简单进行物理混合, 无法产生 更进一步的相互作用. 为此, 徐铜文组 ${ }^{[84]}$ 通过兴斯堡反 应, 将 Fe-MIL-101- $\mathrm{NH}_{2}$ 晶体链接到磺化聚苯醚(SPPO) 链上, 消除两相界面之间的影响以制备均一的复合膜 SPPO-MOFs. 该复合膜在 $90{ }^{\circ} \mathrm{C}, 98 \% \mathrm{RH}$ 条件下的质子 电导率为 $0.25 \mathrm{~S} \cdot \mathrm{cm}^{-1}$, 且经交联后的复合膜热稳定性、 含水率与抗氧化能力都有不同程度的提高. 他们提出复 合膜的质子传递机理是两种机理的共同作用: Fe-MIL-101- $\mathrm{NH}_{2}$ 中的 Fe 离子起到催化水分子解离, 生 成质子的作用, 质子在水分子构建的氢键网络进行传 递, 符合 Grotthuss 机理; 而复合膜基体同时也扮演质子 供体的作用，这类质子首先通过氢键传递至 MOFs 表面， 而后通过水分子作为载体运输到邻近的其它 MOFs, 符 合 Vehicular 机理.

静电纺丝法是指带电荷的高分子溶液或熔体, 在高 压电场的作用下喷射并延展成纤维的过程. 静电纺丝法 制备的纳米纤维比表面积大、孔隙率高、孔径交联, 使 其在膜科学领域受到广泛关注. 特别是质子交换膜应用 方面, 高度取向性的一维纳米纤维结构可被视为天然的 质子传递通道 ${ }^{[85,86]}$. 徐铜文组 ${ }^{[87]}$ 尝试将以氨基-三唑为 配体的立方形 Zn-MOF ZCCH 与磺酰氯化聚醚砜酮 (SPPESK-Cl)进行化学键合, 随后通过静电纺丝得到内 部纤维有序排列的复合膜结构(图 $12 \mathrm{a} \sim 12 \mathrm{~d}$ ). 因 $\mathrm{ZCCH}$ 的粒径小于 $100 \mathrm{~nm}$, 其被直径为约 $200 \mathrm{~nm}$ 的 SPPESK 纤维紧密包裹在内, 有效防止甲醇燃料的泄露. 三唑质 子导体的存在使得该复合膜在 $160{ }^{\circ} \mathrm{C}$ 无水条件下展现 出优异的质子电导率 $\left(8.2 \times 10^{-2} \mathrm{~S} \cdot \mathrm{cm}^{-1}\right)$, 较纤维无序排 列的膜结构有大幅提高, 这是因为三唑与磺酰亚胺在 SPPESK 纳米纤维内部形成了规整的传递路径, 使质子 按 Vehicular 机理进行传导(图 12e).

4.2.2 一维/二维 MOFs 结构作为增强相的混合基质质 子交换膜

采用一维或二维 MOFs 纳米结构, 不仅提高了 MOFs 的比表面积以增强质子扩散, 同时可避免粉体的 团聚. Wang 等 ${ }^{[61]}$ 创造性地制备出具有花瓣状形貌的
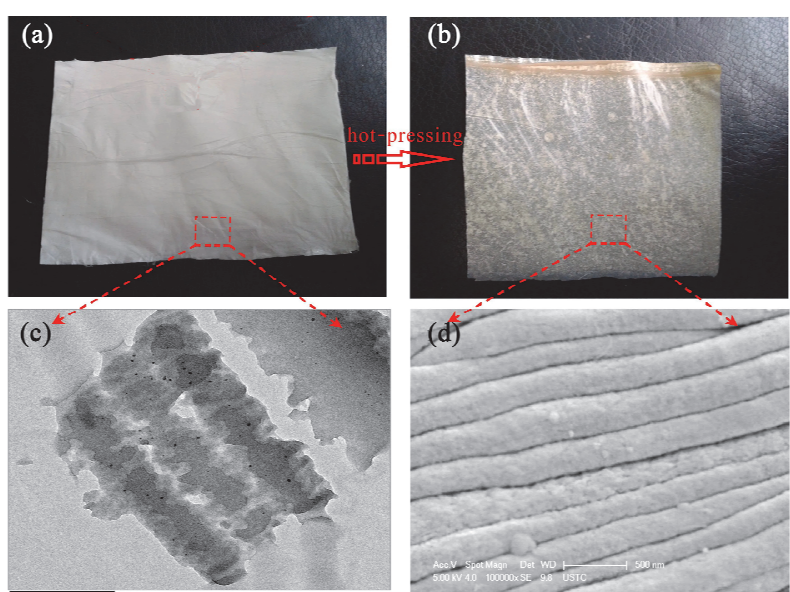

(e)

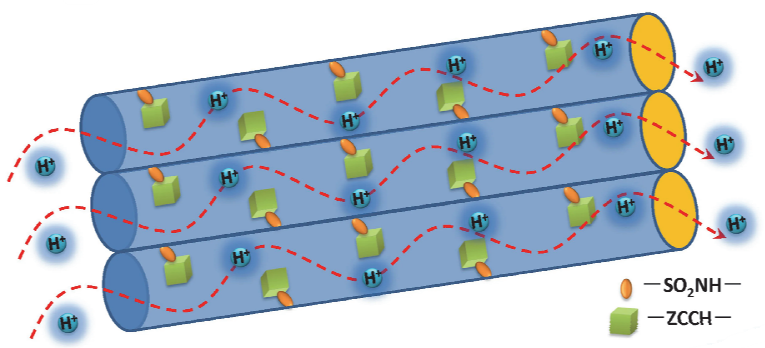

图 $12 \mathrm{SPPESK} / \mathrm{ZCCH}$ 电纺复合膜的光学照片: (a) 热压前与(b)热压 后; SPPESK/ZCCH 复合膜(c)热压前的 TEM 图像与(d) 热压后的. e) SPPESK/ZCCH 质子传递机制示意图

Figure 12 Photos of SPPESK/ZCCH composite membranes: (a) before hot pressing and (b) after hot pressing. (c) TEM of electrospun fiber mats. (d) Surface SEM of electrospun fiber mats after hot-pressing. (e) Schematic illustration of proton conducting mechanism in SPPESK/ZCCH.

MIL-53( $\mathrm{Al}) \mathrm{NH}_{2}$ 纳米纤维 (MNFs) 作为 SPES 纳米纤维的 增强体(图 13a). 当 MNFs 的质量分数为 $5 \%$ 时复合膜的 质子电导率达到 $0.201 \mathrm{~S} \cdot \mathrm{cm}^{-1}\left(80{ }^{\circ} \mathrm{C}, 100 \% \mathrm{RH}\right)$, 同时 强度也提升至 $33.42 \mathrm{MPa}$, 达到了同时增强质子交换性 能和力学性能的效果(图 $13 \mathrm{~b}$ 与 13c).

刘毅组通过水热法合成了一种二维 Ni-BDC 纳米 片, 并加入 PAN 溶液进行静电纺丝得到 Ni-BDC-PAN 复 合膜, 随后分别吸附磷酸 ${ }^{[64]}$ 与咪唑 ${ }^{[63]}$ 制备出 $\mathrm{H}_{3} \mathrm{PO}_{4} @ \mathrm{Ni}-\mathrm{BDC}-\mathrm{PAN}$ 与 Im@Ni-BDC-PAN(图 13d)复合 质子交换纤维膜. 由于 PAN 纳米纤维的高比表面积与 纳米片的高分散性, Ni-BDC 的负载质量分数最高可以 达到 $60 \%$, 且均匀生长在纤维表面, 远超过一般 MMMs 的 MOFs 负载量. Im $@ N i-B D C-P A N$ 膜与 $\mathrm{H}_{3} \mathrm{PO}_{4} @$ $\mathrm{Ni}-\mathrm{BDC}-\mathrm{PAN}$ 的质子电导率分别达到 $1.05 \times 10^{-2} \mathrm{~S} \cdot \mathrm{cm}^{-1}$ $\left(80{ }^{\circ} \mathrm{C}, 90 \% \mathrm{RH}\right)$ 与 $6.04 \times 10^{-5} \mathrm{~S} \cdot \mathrm{cm}^{-1}\left(90{ }^{\circ} \mathrm{C}, 90 \% \mathrm{RH}\right)$.

\subsection{3 含第三相的 MOFs 复合质子交换膜}

在本综述第三节已经提到, 向 MOFs 引入其它具有 质子导电性的物质是提高其质子传递能力的一项常用 策略. 与之相似，向质子交换膜掺入其它客体分子(酸 类物质、咪唑类物质、离子液体等)也被常用于提升膜 的质子电导率. 


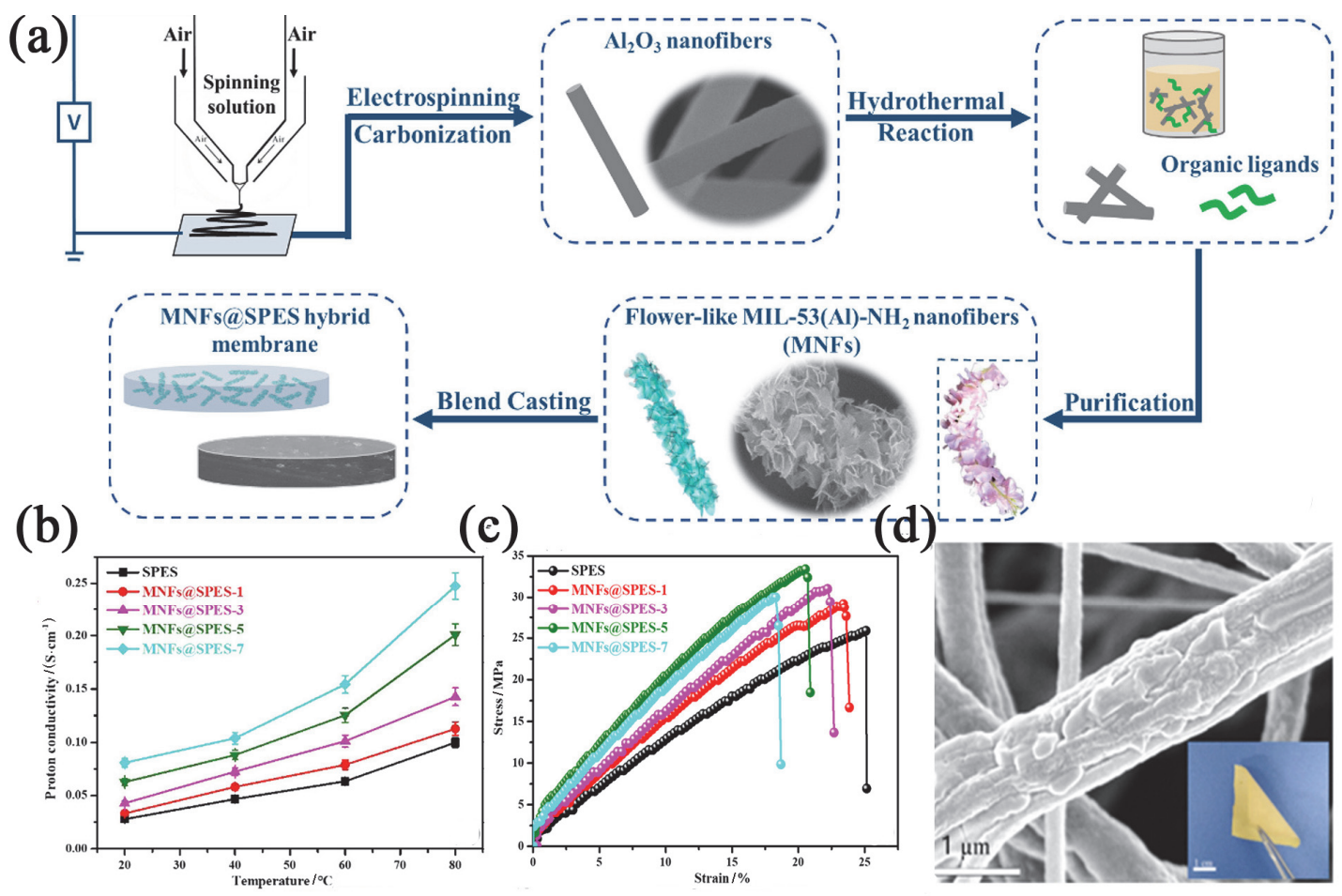

图 13 MNFs@SPES 复合质子交换膜的(a)制备流程, (b) 质子电导率与(c)拉伸强度 ${ }^{[61]}$; (d) Ni-BDC 负载量为 $60 \%$ (质量分数)时的 Im@Ni-BDC-PAN 的 SEM 图像 ${ }^{[63]}$

Figure 13 (a) Schematic illustration of the preparation process of MNFs@SPES, (b) temperature-dependent proton conductivity and (c) tensile strength of MNFs@SPES with different MNFs amount ${ }^{[61]}$, (d) SEM image of 60\% Im@Ni-BDC-PAN ${ }^{[63]}$.

氧化石墨烯(Graphene Oxide, GO)表面的含氧基团 可起到质子供体与质子受体的双重作用, 使得其在质子 交换领域, 特别是高温低湿/无水环境的应用受到广泛 关注 ${ }^{[88,89]}$. 伍斌 ${ }^{[00]}$ 成功在 $\mathrm{GO}$ 片层中原位生长含三唑结 构的 MOF ZCCH, 并通过化学反应与 SPPO 基体键合形 成复合质子交换膜. $\mathrm{GO}$ 在复合膜内部与 $\mathrm{ZCCH}$ 及 $\mathrm{SPPO}$ 形成插层结构, 使得沿 $\mathrm{GO}$ 平面与垂直 $\mathrm{GO}$ 平面同时形 成质子传递通道, 呈现多维性. 该复合膜在无水条件下 质子电导率在 $30 \sim 160{ }^{\circ} \mathrm{C}$ 的范围内逐渐升高, 最高达 到 $0.12 \mathrm{~S} \cdot \mathrm{cm}^{-1}$, 使其在中高温环境下显示巨大的应用 前景.

武培怡课题组细致研究了 GO 同 MOFs 的协同作用 对复合质子交换膜性能的影响. 他们通过原位合成法制 备了一系列 $\mathrm{GO}$ 增强的复合质子交换膜, 包括 ZIF-8@GO/Nafion ${ }^{[65]}, \quad$ GO@UiO-66- $\mathrm{NH}_{2} / \mathrm{Nafion}^{[62]}$, SPEEK/S-UiO-66@GO ${ }^{[91]}$ 等. 他们认为 GO 的加入主 要有以下方面的作用(图 14): (1) MOFs 在 GO 表面的原 位生长保证了其在聚合物基体中的高分散性; (2) GO 与 MOFs 之间的相互作用增强了复合膜的保水率, 保证了 复合膜的质子电导率, 特别是低湿度条件下; (3) MOFs@GO 结构与聚合物基体间通过基团相互作用形 成的界面层保证了在无水环境下的质子导电性. ZIF-8@GO/Nafion, GO@UiO-66- $\mathrm{NH}_{2} / \mathrm{Nafion}$, SPEEK/ $\mathrm{S}-\mathrm{UiO}-66 @ \mathrm{GO}$ 的高温质子电导率分别为 $0.28 \mathrm{~S} \cdot \mathrm{cm}^{-1}$ $\left(120{ }^{\circ} \mathrm{C}, 40 \% \mathrm{RH}\right), 3.403 \times 10^{-3} \mathrm{~S} \cdot \mathrm{cm}^{-1}\left(90{ }^{\circ} \mathrm{C}\right.$, 无水)与
$1.657 \times 10^{-2} \mathrm{~S} \cdot \mathrm{cm}^{-1}\left(100{ }^{\circ} \mathrm{C}, 40 \% \mathrm{RH}\right)$ ，且复合膜形稳 性、强度与甲醇阻隔性较基体膜均有明显提高.

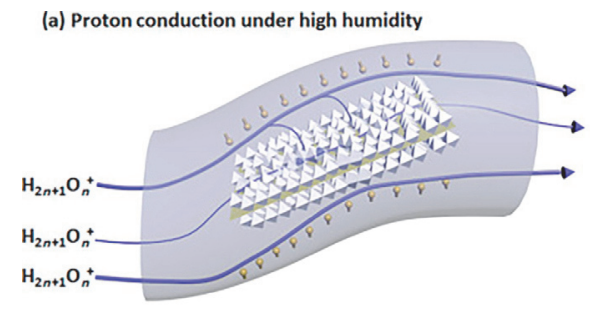

(b) Proton conduction under anhydrous condition
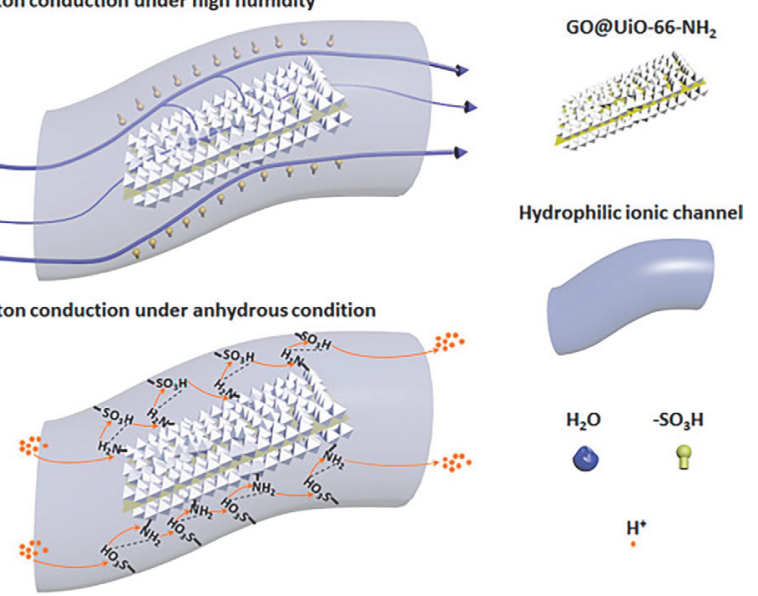

$\mathrm{H}_{2} \mathrm{O}-\mathrm{SO}_{3} \mathrm{H}$

图 14 Polymer/GO@MOF 结构典型的质子传递路径: (a)高湿条件下, (b)无水条件下 ${ }^{[22]}$

Figure 14 Schematic illustration of proton conducting process in a typical polymer/GO@MOF structure: (a) under high humidity and (b) under anhydrous condition ${ }^{[62]}$

除此之外，也有一些使用其它无机纳米颗粒来增强 $\mathrm{MOFs} /$ 聚合物复合质子交换膜性能的工作. 例如, Ahmadian-Alam 等 ${ }^{[92]}$ 向磺化聚砜(mPSU)中添加了含咪唑 的 $\mathrm{NH}_{2} \mathrm{MIL}-53(\mathrm{Al})$ 与磺化硅纳米颗粒 $\left(\mathrm{Si}-\mathrm{SO}_{3} \mathrm{H}\right)$ 制备复 合质子交换膜. 第三相加入使复合膜的拉伸强度由 
19.74 $\mathrm{MPa}$ 提升至 $30.08 \mathrm{MPa}$ ，同时模量由 $6.75 \mathrm{GPa}$ 跃升 至 $14.98 \mathrm{GPa}$. 当 $\mathrm{Si}-\mathrm{SO}_{3} \mathrm{H}$ 的质量分数为 $5 \%$ 时, 复合膜 的质子导电性提升最明显, 达到 $1.7 \times 10^{-2} \mathrm{~S} \cdot \mathrm{cm}^{-1}$ $\left(70{ }^{\circ} \mathrm{C}\right)$, 同时输出功率密度达到 $40.80 \mathrm{~mW} \cdot \mathrm{cm}^{-2}$.

\section{5 总结与展望}

以电动汽车与新型潜艇为代表的科技对燃料电池 的效率提出了更高的要求. 高温质子交换膜燃料电池 (运行温度 $120 \sim 200{ }^{\circ} \mathrm{C}$ ) 反应速率高, 燃料扩散快, 无需 大量水系统, 是满足上述应用的理想选择. 然而, 常用 的 Nafion 系列质子交换膜性能在超过 $80{ }^{\circ} \mathrm{C}$ 时便显著下 降. MOFs 因其高孔隙率、结构多样、制备简便等特性, 在质子交换膜领域具有很强的发展潜力. MOFs 进行质 子传递主要依靠质子跳跃机制与质子运输机制两种模 式进行. 研究者们通过结构修饰与引入客体分子等手段 不断提升 MOFs 的质子导电性, 一些 MOFs 的电导率甚 至超过了 $10^{-2} \mathrm{~S} \cdot \mathrm{cm}^{-1}$. 同时, MOFs 质子交换膜的研究 也取得一些突破和进展. 然而, 必须看到的是将 MOFs 质子交换膜真正应用于燃料电池还有很长一段路要走, 其发展主要受限于以下问题:

(1) 目前, 很多 MOFs 的质子导电性极其依赖水分 子的存在，限制了其在更宽的温度与湿度范围内的应 用. 而已报道的高温、无水条件下应用的 MOFs 质子导 体在室温下导电性又较差, 这是因为客体分子在室温下 无法提供质子导致的. 通过精细设计 MOFs 结构, 使其 内部在无水状态即可形成有效的氢键网络是解决此问 题的一种有效手段, 但是目前针对这方面的研究甚少. 开发既能在室温、高湿环境, 又能在中高温、低湿下保 持较好质子导电性的 MOFs 质子导体将大幅拓宽其应用 领域.

(2) MOFs 在复合膜基体中的质子传递机理尚不能 完全研究清楚. 尽管研究者们尝试利用研究纯 MOFs 质 子导体的方式揭露 MOFs 复合质子交换膜的工作机理, 但因为两者的工作环境不同, 其数据准确度存在较大的 差异. 同时，晶界效应、界面配合度等因素极大程度影 响复合膜质子交换性能的发挥, 深刻认识 MOFs 与基体 间的相互作用将对设计制备高性能复合质子交换膜产 生巨大影响.

（3）尽管一些基于 MOFs 的质子交换膜具有优异的 性能, 但只有少部分工作考察了其在电池环境的工作状 态, 特别是在高温(120 200 $\left.{ }^{\circ} \mathrm{C}\right)$ 环境下的表现与全电 池测试报道接近空白. 如何选用具有耐高温特性的基 体, 并通过掺杂合适的 MOFs 尽可能提高其质子导电性, 是该领域目前亟待研究的方向.

(4) 二维 MOFs 纳米材料兼顾了 MOFs 的高孔隙率 与二维材料的高比表面积、优异物理化学性能等特征, 具有广阔的应用前景. 然而, 目前关于二维 MOFs 纳米 结构的质子导电性的研究几乎没有, 同时关于 MOFs 质
子导体薄膜的研究也很少. 可以预测二维 MOFs 纳米质 子导体材料会有可观的性能，且可能与 MOFs 质子导体 薄膜一起开拓该领域的新发展空间.

\section{作者简介}

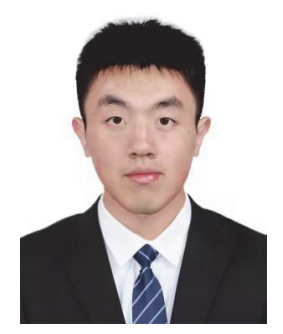

孙炼, 2018 年毕业于国防科技大学材料科学与工程专业, 获工学硕士学位. 现为国防科技大学在读博士, 导师周新贵 教授. 主要从事金属有机框架材料及质子交换膜相关研究.

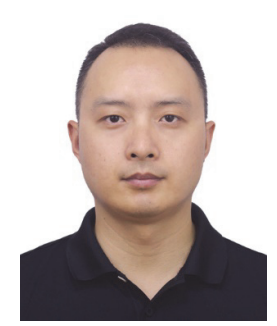

王洪否, 副教授, 1983 年生, 2012 年毕业于国防科技大学 并获得材料科学与工程博士学位, 2015 2018 年在中国工程 物理研究院开展博士后研究工作, 2019 年 10１2 月在伦敦玛 丽女王大学开展短期访问. 主要从事陶瓷基复合材料研究工 作.

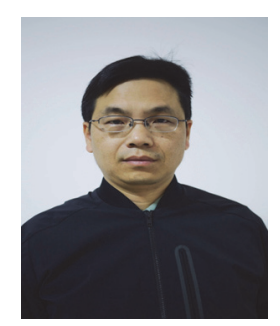

余金山, 1973 年生, 2006 年毕业于上海交通大学并获得材 料学博士学位, 随后在日本东北大学从事 3 年博士后研究工 作, 2009 年回国后在国防科技大学任副研究员, 从事陶瓷基复 合材料制备工艺研究及材料分析测试方面的工作.

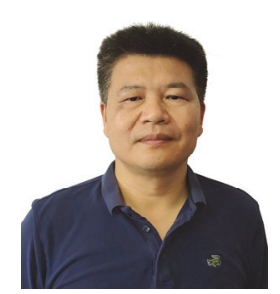

周新贵, 1968 年生, 国防科技大学空天科学学院教授, 博 士生导师. 2006 年在中南大学获得工学博士学位, 2008 年在布 里斯托大学访学一年. 主要研究方向为陶瓷基复合材料以及 功能陶瓷，主持参与 15 项国家重点科研项目，荣获国家科技 
进步二等奖、省部级科技进步一等奖、全国发明展金奖. 发表 学术论文 90 余篇, 其中 70 篇为 SCI 检索.

\section{References}

[1] Escorihuela, J.; Narducci, R.; Compañ, V.; Costantino, F. Adv. Mater. Interfaces 2019, 6, 1801146.

[2] Haubold, H. G.; Vad, T.; Jungbluth, H.; Hiller, P. Electrochim. Acta 2001, 46, 1559.

[3] Jaafar, J.; Nordin, M.; Hadi, N. A.; Ismail, A. F.; Othman, M. H. D.; A Rahman, M.; Aziz, F. J. Membr. Sci. Res. 2019, 5, 65.

[4] Fang, J.; Shen, P. K. J. Membr. Sci. 2006, 285, 317.

[5] Zhang, Y.; Zheng, L.; Liu, B.; Wang, H.; Shi, H. J. Membr. Sci. 2019, 584, 173.

[6] Chen, Z. Y.; Liu, J. W.; Cui, H.; Zhang, L.; Su, C. Y. Acta Chim. Sinica 2019, 77, 242 (in Chinese). (陈之尧, 刘捷威, 崔浩, 张利, 苏成勇, 化学学报, 2019, 77, 242.)

[7] Qiao, W.; Song, T.; Zhao, B. Chin. J. Chem. 2019, 37, 474.

[8] Dai, M. M.; Wang, J.; Li, L. G.; Wang, Q.; Liu, M. N.; Zhang, Y. G. Acta Chim. Sinica 2020, 78, 355 (in Chinese). (代迷迷, 王健, 李麟 阁，王琪，刘美男，张跃钢，化学学报, 2020, 78, 355.)

[9] He, T.; Zhang, Y.-Z.; Wu, H.; Kong, X.-J.; Liu, X.-M.; Xie, L.-H.; Dou, Y.; Li, J.-R. ChemPhysChem 2017, 18, 3245.

[10] Kanda, S.; Yamashita, K.; Ohkawa, K. Bull. Chem. Soc. Jpn. 1979, $52,3296$.

[11] Shimizu, G. K. H.; Taylor, J. M.; Kim, S. Science 2013, 341, 354.

[12] Ye, Y.; Gong, L.; Xiang, S.; Zhang, Z.; Chen, B. Adv. Mater. 2020, 32, 1907090 .

[13] Lim, D. W.; Kitagawa, H. Chem. Rev. 2020, 120,8416

[14] Li, W.-H.; Deng, W.-H.; Wang, G.-E.; Xu, G. EnergyChem 2020, 2, 100029.

[15] Agmon, N. Chem. Phys. Lett. 1995, 244, 456

[16] Kreuer, K. D.; Rabenau, A.; Weppner, W. Angew. Chem. Int. Ed. 1982, 21, 208.

[17] Zhang, J.; Bai, H.-J.; Ren, Q.; Luo, H.-B.; Ren, X.-M.; Tian, Z.-F.; Lu, S. ACS Appl. Mater. Interfaces 2018, 10, 28656.

[18] Wang, Z. T.; Li, H.; Yan, S. C.; Fang, Q. R. Acta Chim. Sinica 2020, 78,63 (in Chinese). (王志涛, 李辉, 颜士臣, 方千荣, 化学学报, 2020, 78, 63.)

[19] Umeyama, D.; Horike, S.; Inukai, M.; Itakura, T.; Kitagawa, S. J. Am. Chem. Soc. 2012, 134, 12780.

[20] Liu, M.; Chen, L.; Lewis, S.; Chong, S. Y.; Little, M. A.; Hasell, T.; Aldous, I. M.; Brown, C. M.; Smith, M. W.; Morrison, C. A.; Hardwick, L. J.; Cooper, A. I. Nat. Commun. 2016, 7, 12750.

[21] Zhang, K.; Xie, X.; Li, H.; Gao, J.; Nie, L.; Pan, Y.; Xie, J.; Tian, D.; Liu, W.; Fan, Q. Adv. Mater. 2017, 29, 1701804.

[22] Wu, L.; Yang, Y.; Ye, Y.; Yu, Z.; Song, Z.; Chen, S.; Chen, L.; Zhang, Z.; Xiang, S. ACS Appl. Energy Mater. 2018, 1, 5068.

[23] Bian, L.; Li, W.; Wei, Z. Z.; Liu, X. W.; Li, S. Acta Chim. Sinica 2018, 76, 303 (in Chinese). (市磊, 李炜, 魏振振, 刘晓威, 李松, 化学学报, 2018, 76, 303.)

[24] Yang, F.; Huang, H.; Wang, X.; Li, F.; Gong, Y.; Zhong, C.; Li, J.-R. Cryst. Growth Des. 2015, 15, 5827.

[25] Losch, P.; Joshi, H. R.; Vozniuk, O.; Grünert, A.; Ochoa-Hernández, C.; Jabraoui, H.; Badawi, M.; Schmidt, W. J. Am. Chem. Soc. 2018, $140,17790$.

[26] Sun, Z.; Yu, S.; Zhao, L.; Wang, J.; Li, Z.; Li, G. Chem.-Eur. J. 2018, 24, 10829.

[27] Yamada, T.; Sadakiyo, M.; Kitagawa, H. J. Am. Chem. Soc. 2009, 131,3144

[28] Yang, F.; Xu, G.; Dou, Y.; Wang, B.; Zhang, H.; Wu, H.; Zhou, W.; Li, J.-R.; Chen, B. Nat. Energy 2017, 2, 877.

[29] Tang, Q.; Yang, Y.-L.; Zhang, N.; Liu, Z.; Zhang, S.-H.; Tang, F.-S.; Hu, J.-Y.; Zheng, Y. Z.; Liang, F. P. Inorg. Chem. 2018, 57, 9020.

[30] Wu, H.; Yang, F.; Lv, X. L.; Wang, B.; Zhang, Y.-Z.; Zhao, M. J.; Li, J. R. J. Mater. Chem. A 2017, 5, 14525.

[31] Feng, L.; Wang, H. S.; Xu, H. L.; Huang, W. T.; Zeng, T. Y.; Cheng, Q. R.; Pan, Z. Q.; Zhou, H. Chem. Commun. 2019, 55, 1762.

[32] Zhang, F.-M.; Dong, L.-Z.; Qin, J.-S.; Guan, W.; Liu, J.; Li, S.-L.; Lu, M.; Lan, Y. Q.; Su, Z. M.; Zhou, H. C. J. Am. Chem. Soc. 2017, $139,6183$.

[33] Horike, S.; Chen, W.; Itakura, T.; Inukai, M.; Umeyama, D.; Asakura, H.; Kitagawa, S. Chem. Commun. 2014, 50, 10241.

[34] Liu, L.; Yao, Z.; Ye, Y.; Liu, C.; Lin, Q.; Chen, S.; Xiang, S.; Zhang, Z. ACS Appl. Mater. Interfaces 2019, 11, 16490.
[35] Liu, R.; Zhao, L.; Yu, S.; Liang, X.; Li, Z.; Li, G. Inorg. Chem. 2018, 57, 11560 .

[36] Chen, H.; Han, S. Y.; Liu, R. H.; Chen, T. F.; Bi, K. L.; Liang, J. B.; Deng, Y. H.; Wan, C. Q. J. Power Sources 2018, 376, 168.

[37] Meng, X.; Wei, M.-J.; Wang, H. N.; Zang, H. Y.; Zhou, Z. Y. Dalton Trans. 2018, 47, 1383.

[38] Gui, D.; Dai, X.; Tao, Z.; Zheng, T.; Wang, X.; Silver, M. A.; Shu, J.; Chen, L.; Wang, Y.; Zhang, T. J. Am. Chem. Soc. 2018, 140, 6146.

[39] Shigematsu, A.; Yamada, T.; Kitagawa, H. J. Am. Chem. Soc. 2011, 133, 2034.

[40] Sarango-Ramírez, M. K.; Lim, D.-W.; Kolokolov, D. I.; Khudozhitkov, A. E.; Stepanov, A. G.; Kitagawa, H. J. Am. Chem. Soc. 2020, 142, 6861.

[41] Bao, S. S.; Shimizu, G. K.; Zheng, L. M. Coord. Chem. Rev. 2019, $378,577$.

[42] Taylor, J. M.; Mah, R. K.; Moudrakovski, I. L.; Ratcliffe, C. I.; Vaidhyanathan, R.; Shimizu, G. K. H. J. Am. Chem. Soc. 2010, 132 , 14055.

[43] Taylor, J. M.; Dawson, K. W.; Shimizu, G. K. H. J. Am. Chem. Soc. 2013, 135, 1193.

[44] Ramaswamy, P.; Wong, N. E.; Gelfand, B. S.; Shimizu, G. K. H. J. Am. Chem. Soc. 2015, 137, 7640.

[45] Luo, Y. H.; Yi, L. Q.; Lu, J. N.; Dong, L.-Z.; Lan, Y. Q. CrystEngComm 2018, 20, 6077.

[46] Li, X. M.; Dong, L. Z.; Li, S. L.; Xu, G.; Liu, J.; Zhang, F. M.; Lu, L. S.; Lan, Y. Q. ACS Energy Lett. 2017, 2, 2313.

[47] Li, R.; Wang, S. H.; Chen, X. X.; Lu, J.; Fu, Z. H.; Li, Y.; Xu, G.; Zheng, F. K.; Guo, G. C. Chem. Mater. 2017, 29, 2321.

[48] Nagarkar, S. S.; Unni, S. M.; Sharma, A.; Kurungot, S.; Ghosh, S. K. Angew. Chem. 2014, 126, 2676.

[49] Hurd, J. A.; Vaidhyanathan, R.; Thangadurai, V.; Ratcliffe, C. I.; Moudrakovski, I. L.; Shimizu, G. K. H. Nat. Chem. 2009, 1, 705.

[50] Bureekaew, S.; Horike, S.; Higuchi, M.; Mizuno, M.; Kawamura, T.; Tanaka, D.; Yanai, N.; Kitagawa, S. Nat. Mater. 2009, 8, 831.

[51] Ye, Y.; Guo, W.; Wang, L.; Li, Z.; Song, Z.; Chen, J.; Zhang, Z.; Xiang, S.; Chen, B. J. Am. Chem. Soc. 2017, 139, 15604.

[52] Sun, X. L.; Deng, W. H.; Chen, H.; Han, H. L.; Taylor, J. M.; Wan, C. Q.; Xu, G. Chem.-Eur. J. 2017, 23, 1248.

[53] Hermes, S.; Schröder, F.; Chelmowski, R.; Wöll, C.; Fischer, R. A. J. Am. Chem. Soc. 2005, 127, 13744.

[54] Xu, G.; Otsubo, K.; Yamada, T.; Sakaida, S.; Kitagawa, H. J. Am. Chem. Soc. 2013, 135, 7438.

[55] Kim, S.; Wang, H.; Lee, Y. M. Angew. Chem. Int. Ed. 2019, 58, 17512.

[56] Dechnik, J.; Gascon, J.; Doonan, C. J.; Janiak, C.; Sumby, C. J. Angew. Chem. Int. Ed. 2017, 56, 9292.

[57] Niluroutu, N.; Pichaimuthu, K.; Sarmah, S.; Dhanasekaran, P.; Shukla, A.; Unni, S. M.; Bhat, S. D. New J. Chem. 2018, 42, 16758.

[58] Guo, Y.; Jiang, Z.; Ying, W.; Chen, L.; Liu, Y.; Wang, X.; Jiang, Z.-J.; Chen, B.; Peng, X. Adv. Mater. 2018, 30, 1705155.

[59] Cai, Y. Y.; Yang, Q.; Zhu, Z. Y.; Sun, Q. H.; Zhu, A. M.; Zhang, Q G.; Liu, Q. L. J. Membr. Sci. 2019, 590, 117277.

[60] Han, R.; Wu, P. ACS Appl. Mater. Interfaces 2018, 10, 18351.

[61] Wang, L.; Deng, N.; Wang, G.; Ju, J.; Cheng, B.; Kang, W. ACS Appl. Mater. Interfaces 2019, 11, 39979.

[62] Rao, Z.; Feng, K.; Tang, B.; Wu, P. J. Membr. Sci. 2017, 533, 160.

[63] Bai, Z.; Liu, S.; Chen, P.; Cheng, G.; Wu, G.; Liu, Y. Nanotechnology 2020, 31, 125702.

[64] Bai, Z.; Liu, S.; Cheng, G.; Wu, G.; Liu, Y. Micropor. Mesopor. Mat. 2020, 292, 109763.

[65] Yang, L.; Tang, B.; Wu, P. J. Mater. Chem. A 2015, 3, 15838.

[66] Ru, C.; Gu, Y.; Na, H.; Li, H.; Zhao, C. ACS Appl. Mater. Interfaces 2019, 11, 31899.

[67] Zhang, F.; Zhang, T.; Zou, X.; Liang, X.; Zhu, G.; Qu, F. Solid State Ionics 2017, 301, 125 .

[68] Rao, Z.; Tang, B.; Wu, P. ACS Appl. Mater. Interfaces 2017, 9, 22597.

[69] Dong, X.-Y.; Wang, J.-H.; Liu, S.-S.; Han, Z.; Tang, Q.-J.; Li, F.-F.; Zang, S.-Q. ACS Appl. Mater. Interfaces 2018, 10, 38209.

[70] Adams, R.; Carson, C.; Ward, J.; Tannenbaum, R.; Koros, W. Micropor. Mesopor. Mat. 2010, 131, 13.

[71] Sabetghadam, A.; Liu, X.; Gottmer, S.; Chu, L.; Gascon, J.; Kapteijn, F. J. Membr. Sci. 2019, 570-571, 226.

[72] Liu, Y.; Liu, G.; Zhang, C.; Qiu, W.; Yi, S.; Chernikova, V.; Chen, Z.; Belmabkhout, Y.; Shekhah, O.; Eddaoudi, M.; Koros, W. Adv. Sci. 2018, 5, 1800982 . 
[73] Cao, L.; Tao, K.; Huang, A.; Kong, C.; Chen, L. Chem. Commun. 2013, 49, 8513.

[74] Anjum, M. W.; Vermoortele, F.; Khan, A. L.; Bueken, B.; De Vos, D. E.; Vankelecom, I. F. J. ACS Appl. Mater. Interfaces 2015, 7, 25193.

[75] Ordoñez, M. J. C.; Balkus, K. J.; Ferraris, J. P.; Musselman, I. H. J. Membr. Sci. 2010, 361, 28.

[76] Dorosti, F.; Omidkhah, M.; Abedini, R. Chem. Eng. Res. Des. 2014, 92, 2439.

[77] Duan, L.; Wang, Y.; Zhang, Y.; Liu, J. Appl. Surf. Sci. 2015, 355, 436.

[78] Li, W.; Samarasinghe, S. A. S. C.; Bae, T.-H. J. Ind. Eng. Chem. 2018, 67, 156 .

[79] Ru, C.; Li, Z.; Zhao, C.; Duan, Y.; Zhuang, Z.; Bu, F.; Na, H. ACS Appl. Mater. Interfaces 2018, 10, 7963.

[80] Katz, M. J.; Brown, Z. J.; Colón, Y. J.; Siu, P. W.; Scheidt, K. A.; Snurr, R. Q.; Hupp, J. T.; Farha, O. K. Chem. Commun. 2013, 49, 9449.

[81] Peng, X.; Ye, L.; Ding, Y.; Yi, L.; Zhang, C.; Wen, Z. Appl. Catal., B 2020, 260, 118152 .

[82] Liu, S.; Sang, X.; Wang, L.; Zhang, J.; Song, J.; Han, B. Electrochim. Acta 2017, 257, 243.
[83] Zhang, B.; Cao, Y.; Li, Z.; Wu, H.; Yin, Y.; Cao, L.; He, X.; Jiang, Z. Electrochim. Acta 2017, 240, 186

[84] Wu, B.; Lin, X.; Ge, L.; Wu, L.; Xu, T. Chem. Commun. 2013, 49, 143.

[85] Liu, W.; Wang, S.; Xiao, M.; Han, D.; Meng, Y. Chem. Commun. 2012, 48, 3415 .

[86] Liu, X.; Yang, Z.; Zhang, Y.; Li, C.; Dong, J.; Liu, Y.; Cheng, H. Int. J. Hydrogen Energy 2017, 42, 10275.

[87] Wu, B.; Pan, J.; Ge, L.; Wu, L.; Wang, H.; Xu, T. Sci. Rep. 2014, 4, 4334.

[88] Choi, B. G.; Huh, Y. S.; Park, Y. C.; Jung, D. H.; Hong, W. H.; Park, H. Carbon 2012, 50, 5395.

[89] Enotiadis, A.; Angjeli, K.; Baldino, N.; Nicotera, I.; Gournis, D. Small 2012, 8, 3338.

[90] Wu, B. Ph.D. Dissertation, University of Science and Technology of China, Hefei, 2015 (in Chinese). (伍斌, 博士论文, 中国科学技术 大学, 合肥, 2015).

[91] Sun, H.; Tang, B.; Wu, P. ACS Appl. Mater. Interfaces 2017, 9 , 26077.

[92] Ahmadian-Alam, L.; Mahdavi, H. Renew. Energ. 2018, 126, 630.

(Cheng, B.) 